\title{
Numerical investigation of the effects of a small fixed sphere in tandem arrangement on VIV of a sphere
}

\author{
Syed Ahmad Raza, Ming-Jyh Chern *, Herman Susanto, Yun-Hui Zhou \\ Department of Mechanical Engineering, National Taiwan University of Science and Technology, Taipei, 10607, Taiwan
}

\section{A R T I C L E I N F O}

\section{Keywords:}

Vortex-induced vibration

Lock-in

Direct-forcing immersed boundary method

VIV control

\begin{abstract}
A B S T R A C T
A direct-forcing immersed boundary (DFIB) method with a virtual force is used to investigate the vortex-induced vibration (VIV) of an elastically mounted sphere in uniform flow at a moderate Reynolds number. A novel method is investigated to control VIV of an elastically mounted sphere by placing a small fixed sphere inline in tandem arrangement. The numerical method is validated by comparisons with previously published results for flow past a stationary sphere and for VIV phenomena. The influence of the small sphere diameter and the gap between the spheres on VIV are studied through an analysis of the hydrodynamic force coefficients, sphere responses, vortex shedding modes and energy efficiency. Regression analysis is conducted to propose mathematical relations for estimating the maximum values of hydrodynamic force coefficients and amplitude ratio. This study proves the capability of the DFIB model for investigating VIV of a sphere. It is also found that the small fixed sphere upstream increases the lift coefficient and amplitude ratio of the vibrating sphere while the drag coefficient is reduced. Furthermore, the energy efficiency of VIV of the sphere is found to be increased by up to three times due to the upstream small fixed sphere.
\end{abstract}

\section{Introduction}

\subsection{Vortex-induced vibration}

In science and engineering fields, interaction between fluid and structure is very common. Extensive research on this phenomenon has been conducted in the last decade. Vortex-induced vibration (VIV) is one of many phenomena caused by fluid-structure interactions (FSI). VIV is the oscillatory response generated by unsteady hydrodynamic forces due to the alternate vortices shed downstream of the immersed solid body in fluid flow.

Applications of VIV involve widespread engineering design cases such as structural design in civil engineering, aircraft design in aerospace engineering, development of water transportation in marine and ocean engineering, as well as the development of energy extraction technology. Depending on the application, this phenomenon can be an engineering challenge or a source of renewable energy. Tall narrow structures such as industrial chimneys in close proximity are considered to be at risk from VIV. Recently, various studies have been conducted to develop energy generation devices that utilize vortex from FSI such as Vortex Induced Vibration Aquatic Clean Energy (VIVACE) (Bernitsas et al., 2008;
Bernitsas and Raghavan, 2011) and bladeless aerogenerator vortex. Consequently, it is very useful to conduct further research to provide new insight on how to increase the energy produced by the VIV phenomenon or reduce the impact of damage caused by it.

VIV has the potential to cause major structural failures but also provides an innovative opportunity as renewable energy source. Many studies have been conducted to investigate large-amplitude oscillations to minimize the effects of the flow-induced vibrations to prevent structure failures. VIV is caused by unsteady hydrodynamic forces arising from the alternate vortex shedding behind the solid body immersed in fluid flow. This phenomenon could lead to lock-in/synchronization, which may cause the failure of the structure, especially for the resonance case. Therefore, VIV has led to a large number of fundamental studies in published literature such as Sarpkaya (1979), Bearman (1984), Parkinson (1989) and Williamson and Govardhan (2004). Studies on VIV have been done numerically as well as experimentally, especially for the case of elastically mounted circular cylinder. Some studies that have been done numerically include Willden and Graham (2001), Yamamoto et al. (2004), Violette et al. (2007), Borazjani and Sotiropoulos (2009) and Bourguet et al. (2011). VIV of a circular cylinder has been investigated experimentally by Khalak and Williamson (1997) and Assi et al. (2006). Experimental studies on VIV of a tethered sphere have been conducted by

\footnotetext{
* Corresponding author.

E-mail address: mjchern@mail.ntust.edu.tw (M.-J. Chern).
} 


\begin{tabular}{|c|c|c|c|}
\hline \multicolumn{2}{|c|}{ Nomenclature } & $\mathrm{p}$ & non-dimensional pressure \\
\hline$\ddot{Y}$ & non-dimensional transverse acceleration of sphere & $\mathrm{t}$ & time, $s$ \\
\hline$\dot{Y}$ & non-dimensional transverse velocity of sphere & $t^{*}$ & non-dimensional time \\
\hline $\mathbf{f}^{*}$ & non-dimensional virtual force & $U_{R}^{*}$ & non-dimensional reduced velocity \\
\hline $\mathrm{u}$ & non-dimensional fluid velocity & $U_{\infty}$ & non-dimensional free stream velocity \\
\hline $\mathbf{u}_{s}$ & non-dimensional velocity of solid & $x, y, z$ & three-dimensional Cartesian coordinates \\
\hline $\mathrm{Re}$ & Reynolds number, $u_{s} D / \nu$ & $x_{r}$ & non-dimensional re-circulation length \\
\hline$A_{y}^{*}$ & non-dimensional amplitude ratio in the transverse direction & $x_{\text {gap }}$ & $\begin{array}{l}\text { non-dimensional gap between the rigid body and the small } \\
\text { fixed sphere }\end{array}$ \\
\hline $\mathrm{C}$ & damping coefficient, $\mathrm{N} \cdot \mathrm{s} \cdot \mathrm{m}^{-1}$ & Y & non-dimensional transverse displacement \\
\hline$C_{D}$ & drag force coefficient & $\gamma$ & displacement of the body \\
\hline$C_{L}$ & lift force coefficient & $\begin{array}{l}\chi \\
\ddot{\gamma}\end{array}$ & acceleration of the body \\
\hline $\mathrm{D}$ & non-dimensional diameter of the rigid body (large sphere) & $\chi$ & $\begin{array}{l}\text { acceleration of the Doay } \\
\text { velocity of the body }\end{array}$ \\
\hline$d_{\text {small }}$ & non-dimensional diameter of the small fixed sphere & $\chi$ & \\
\hline $\mathrm{E}$ & energy efficiency & $\eta$ & volume of solid function \\
\hline $\mathrm{F}$ & non-dimensional total force & $\nu$ & kinematic viscosity of fluid, $\mathrm{m}^{2} \cdot \mathrm{s}^{-1}$ \\
\hline$f^{*}$ & frequency ratio & $\rho$ & non-dimensional density \\
\hline$f_{N}$ & non-dimensional natural frequency of structure & $\xi$ & volume of solid function for sub-grid method \\
\hline$f_{V}$ & non-dimensional frequency of vortex shedding & $\zeta$ & non-dimensional structure damping ratio \\
\hline $\mathrm{k}$ & structural stiffness, $\mathrm{N} \cdot \mathrm{m}^{-1}$ & * & intermediate time step level \\
\hline$m^{*}$ & mass ratio & $\mathrm{m}$ & time step level \\
\hline$m_{r}$ & non-dimensional reduced mass & $\mathrm{f}$ & Fluid \\
\hline$m_{s}$ & structural mass of solid, $\mathrm{kg}$ & $\mathrm{s}$ & Solid \\
\hline $\mathrm{N}$ & number of grids & rms & root mean square value \\
\hline $\mathrm{n}$ & number of sub-grids & & \\
\hline
\end{tabular}

Govardhan and Williamson (1997) and Govardhan and Williamson (2005). Govardhan and Williamson (2005) also investigated a sphere constrained to move only in the transverse direction. Williamson and Govardhan (2004) and Sarpkaya (2004) have conducted comprehensive reviews on such VIV studies.

In addition, research on similar phenomena for two tandem circular cylinders has also been widely conducted, which is more commonly known by wake-induced vibration (WIV). In contrast with VIV, where the vibrations are only caused by the vortex shedding behind the solid body, the WIV phenomenon has an additional force caused by vortex shedding in front of the solid body (Assi, 2014). Alam et al. (2003) did an experimental study of fluctuating fluid forces acting on two circular cylinders in tandem arrangement at a sub-critical Reynolds number. Based on their conclusions, placement of a cylinder of same size in front of another cylinder can increase the fluctuating lift and drag coefficient values of the downstream cylinder by two times or more than those for a single cylinder. Similar to the experimental investigations of Alam et al. (2003), Mysa et al. (2016) conducted a numerical study of the wake-induced vibrations for two tandem circular cylinders at low Reynolds number by varying the upstream cylinder diameter. Their study also shows a larger value of lift coefficient and amplitude ratio when an object of similar size is placed in front of a solid body. Nevertheless, the more complex, three-dimensional problem of a freely oscillating sphere in a flow has been discussed in only a few investigations.

Govardhan and Williamson (2005) carried out an experimental study on VIV of a sphere. In their study, they varied the mass ratio $m^{*}$ for moderate Reynolds numbers between 2000 and 12,000. Based on their work, the amplitude ratio in the lock-in regime is found to increase when the mass-damping parameter is decreased. At the lower value of $\mathrm{Re}=$ 300 , Behara et al. (2011) conducted a numerical investigation for VIV of an elastically mounted sphere with three degrees of freedom and studied the hysteresis and vortex shedding modes. This study is performed by varying reduced velocity $U_{R}^{*}=4-6.9$ to systematically investigate the effect on the ensuing three-dimensional vibrations. The authors find that, depending upon the excitation mechanism, two different modes of vortex shedding can emerge for the same value of $U_{R}^{*}$, namely the hairpin and the spiral modes. Subsequently, Behara and Sotiropoulos (2016) investigated the effects of Reynolds number $(\operatorname{Re}=300-1000)$ and reduced velocity on the modal transition from spiral to hairpin modes.

Investigations on VIV of sphere in the low Reynolds numbers range ( $R e=300$ to 800 ) has also been done by Rajamuni et al. (2018b). Unlike the flow configurations of Behara et al. (2011) and Behara and Sotiropoulos (2016) at $\operatorname{Re}=300$, the sphere in the work of Rajamuni et al. (2018b) is only allowed to move freely in the transverse direction. The response curve in this study was also similar to that observed by Behara et al. (2011) for similar Reynolds number and similar mass ratio $\left(m^{*}=\right.$ 3.8197 ) in the reduced velocity range of $U_{R}^{*}=4-6.9$. Furthermore, Rajamuni et al. (2019) continued their study to make a comparison of the response of three degrees of freedom and one degree of freedom systems at a higher Reynolds number $(\operatorname{Re}=2000)$. They observed that the maximum amplitude for three degree-of-freedom (DOF) was 0.6 diameters but for 1-DOF, it was 0.8 diameters.

Researchers have investigated different ways of controlling the flow over a rigidly mounted body. Several passive and active control techniques that usually modify some part of the body geometry or provide it some forced oscillations have been reviewed by Choi et al. (2008). Similarly, researchers have also used novel methods to control VIV of a sphere, especially the use of rotating spheres. Sareen et al. (2018) investigated the effects of sphere rotation on VIV experimentally while Rajamuni et al. (2018a) conducted a similar study numerically. Both of them found that VIV could be completely suppressed at higher rotation rates. McQueen et al. (2018) conducted experiments to study the effect of sinusoidal sphere rotations on its VIV and incorporated a closed-loop controller to optimize the direction of rotation for minimizing VIV. Later, they conducted a detailed investigation into the response of the sphere using an imposed feedback control (McQueen et al., 2020). Chen et al. (2020) conducted experiments to study VIV suppression of a cylinder using a passive-jet flow control method while Ishihara and Li (2020) conducted a numerical investigation of VIV suppression of a cylinder using helical wires.

There is an opportunity to investigate the response of the sphere in the wake of a smaller rigid-body upstream. This could be another method 
to control the VIV of a sphere, which is explored in the present work.

\subsection{Direct-forcing immersed boundary method}

The immersed boundary (IB) method is an emerging simulation technique for analyzing the interaction between fluid and structure. The immersed boundary method was first introduced by Peskin (1972). This method represents the fluid with an Eulerian grid and the structure with a Lagrangian grid and uses a Dirac delta function as a separator between the two. This method was introduced as an alternative to the more widely used body-fitted methods. A major advantage of this method in comparison to the body-fitted method is that with IB a higher numerical scheme can be applied as it uses the much simpler Cartesian grid. Moreover, this method does not require re-meshing at each time step for simulation of moving objects. Cartesian grids also offer a much better orthogonality with respect to body-fitted methods, which improves the stability of the solution as well as its accuracy. Nevertheless, for objects with more complex geometries, body-fitted methods are easier to use.

Mohd-Yusof (1996) introduced an alternative IB method called the direct-forcing method. This method uses a forcing term determined by the difference between the interpolated velocities on the boundary points and the desired boundary velocities. Instead of using a velocity interpolation to distribute the force from a Lagrangian grid to an Eulerian grid, Chern et al. (2014) used the so-called volume of solid (VOS) function to link the force in the FSI. Later, a function of $\eta$ was introduced by Chern et al. (2015) to represent the volume fraction of solid in a cell. $\eta=$ 1 if the grid cell is occupied by the solid, whereas it is zero if it is filled with fluid. A virtual force term ( $\left.\mathbf{f}^{*}\right)$ is used in the momentum equation to account for the effects of FSI.

\subsection{Present study}

The present work uses the same methodology as Chern et al. (2015) for simulation of FSI to study the use of a small fixed sphere upstream inline in tandem arrangement for controlling the VIV of a sphere. The effect of the size of smaller sphere and the gap between them is also investigated. A finer grid size is applied around the solid object. Firstly the three-dimensional flow past a sphere is studied to validate the in-house developed solver. Next the transverse vibration of the sphere is investigated to compare with published results of VIV phenomena for spheres. Finally, a smaller fixed sphere is placed upstream of the transversely vibrating sphere to study whether the size of the smaller sphere and the gap between the two can be used control VIV of the larger sphere.

The in-house solver was developed in $\mathrm{C}++$. Parallelization using OpenMP and a non-uniform grid are used to reduce the computational time. Simulations were run using Dell T630 workstations with 20 cores each consisting of an Intel Xeon CPU E5-2650v3 (2.30 GHz).

The next section will describe the adopted numerical method and mathematical models. The third section will present the validation and numerical sensitivity studies as well as the main findings of the present work. The last section discusses conclusions and future possibilities.

\section{Mathematical and numerical model}

Three kinds of cases are investigated in the present study. The first case is three-dimensional flow past a sphere. This case is considered for validation of the in-house developed computational solver in $\mathrm{C}++$. The second case is the transverse vibration of a sphere to compare the computational solver results for VIV phenomenon of a sphere with previously published literature. The parameters that are compared with previous studies include amplitude ratio $\left(A_{y}^{*}\right)$, frequency ratio $\left(f^{*}\right)$, the hydrodynamic force coefficients $\left(\overline{C_{D}}\right.$ and $\left.C_{L, \text { rms }}\right)$ and the vortex shedding modes. Finally the main problem of the present investigation is discussed, which is a sphere's transverse vibration behind a smaller fixed sphere, as explained visually in Fig. 1. A detailed analysis is conducted by varying the diameter of the smaller fixed sphere and the gap between the two spheres. The parameters considered for investigation are amplitude ratio $\left(A_{y}^{*}\right)$, frequency ratio $\left(f^{*}\right)$, hydrodynamic force coefficients $\left(\overline{C_{D}}\right.$ and $C_{L, \mathrm{rms}}$ ), as well as energy efficiency $(E)$ and vortex shedding modes.

Handling complex geometries while maintaining a sufficiently fast execution time is highly desirable in computational fluid dynamics. The immersed boundary method can use simple Cartesian grids to model complex geometries and thus, avoid the time spent on re-meshing about a moving body at every timestep. In the present study, a type of directforcing method is utilized to establish the proposed immersed boundary of the sphere(s). In order to solve the interaction between fluids and solids, a virtual force is added to incompressible Navier-Stokes equations, which are solved using the finite volume method.

This work uses direct-forcing immersed boundary (DFIB) in the same manner as presented by Chern et al. (2015) and Chern et al. (2018), which has been successfully utilized for investigation of FSI including VIV problems. This will be explained further in section 2.2. For analysis of VIV phenomena, the approach adopted by Govardhan and Williamson (2005) and Chern et al. (2012) has been used.

\subsection{Governing equations}

The continuity eq. (1), momentum eq. (2) and rigid-body motion eq. (3) are utilized. The non-dimensionalized governing equations for an incompressible Newtonian fluid can be expressed as follows:

$$
\nabla \cdot \mathbf{u}=0
$$

$$
\frac{\partial \mathbf{u}}{\partial t^{*}}+\nabla \cdot(\mathbf{u u})=-\nabla p+\frac{1}{\operatorname{Re}} \nabla^{2} \mathbf{u}+\mathbf{f}^{*}
$$

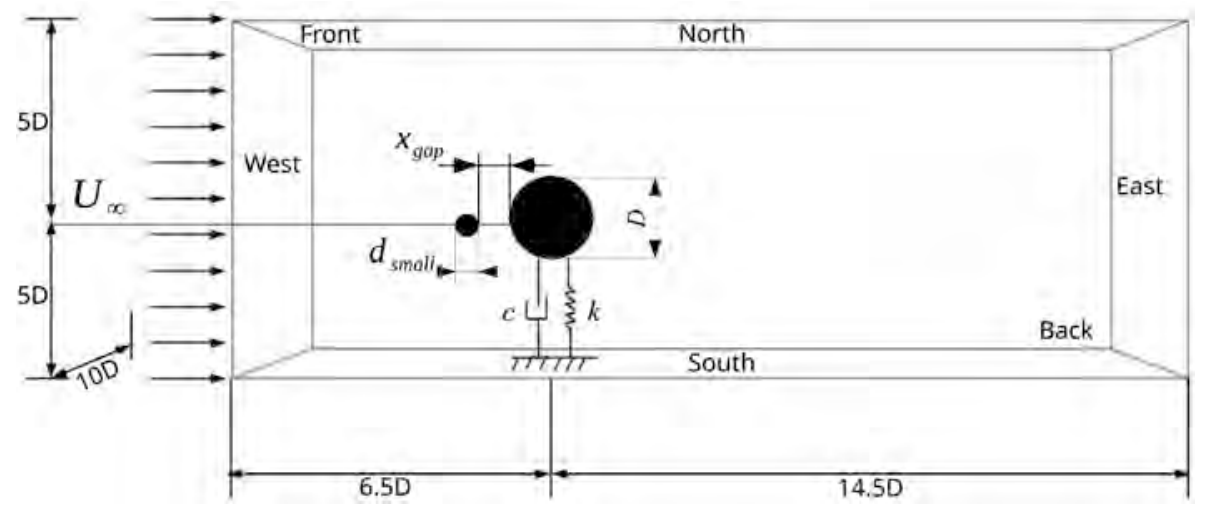

Fig. 1. Schematic of three-dimensional flow past an oscillating sphere downstream of a small fixed sphere. This figure is also applicable for the cases of a single fixed sphere (without vibration and without an upstream smaller sphere) and a single vibrating sphere (without smaller upstream sphere). 
$m_{s} \ddot{\chi}+c \dot{\chi}+k \chi=F(t)$

where $\mathbf{u}, t^{*}$, and $p$ represent the fluid flow velocity, time and pressure, respectively. Reynolds number (Re) is given by $U_{\infty} D / \nu$, where $U_{\infty}$ is the inlet velocity, $D$ is the sphere diameter (large sphere subject to VIV phenomenon) and $v$ is the kinematic viscosity of fluid. $\mathbf{f}^{*}$ represents the virtual force applied only to the solids. $m_{s}, c, k$ and $F$ in the rigid-body motion eq. (3) represent mass of the solid body, the damping coefficient, the spring constant and the total force as a function of time, respectively, while $\chi, \dot{\chi}$ and $\ddot{\chi}$ indicate the displacement, velocity and acceleration of the body, respectively.

\subsection{Direct-forcing immersed boundary method}

DFIB method has been used to analyze the interactions between fluid and solid. For an object undergoing oscillations, a large number of grids may be required to resolve its motion accurately using a Cartesian grid. In the present work, this issue is tackled using the subgrid method, which can accurately model the moving solid object without the need to increase grid numbers of the base grid.

In order to distinguish between fluid and solid, the current approach uses a variable, $\eta$, to represent the volume of solid (VOS), which is calculated for every cell in the computational domain. The maximum value of $\eta$ is 1 , which refers to a cell completely occupied by the solid object as shown in cell number 2 in Fig. 2 (b). Conversely, the minimum value of $\eta$ is 0 , which means the cell lies entirely in the fluid region as represented by cell number 1 of Fig. 2(b). Fig. 2(a) visually describes the algorithmic steps that must be undertaken to determine the VOS or $\eta$.

Referring to Fig. $2, D_{m, n, o}$ is the distance measured from the center of a sphere to the center of a cell. If $D_{m, n, o}$ is greater than the radius of the sphere, $R$, then the VOS value of the cell is zero. Conversely, if $D_{m, n, o}$ is less than $R$, the VOS value of the cell is equal to 1 . But both of these conditions can only be applied when the difference between $D_{m, n, o}$ and $R$ is greater than one half the diagonal of a cell (see Fig. 2(a), step number 3 ). If this condition is not fulfilled for a cell then it is possible that the cell lies at the intersection of the solid and fluid domains (cell number 3 and 4 in Fig. 2(b)) in which case, use of the sub-grid method is preferable.

The distance $d_{i, j, k}$ is measured from the center of the sphere to the center of a sub-cell. The same criterion as applied to $D_{m, n, o}$ is also used here. The VOS for sub-cell, represented by $\xi$, is equal to 1 if $d_{i, j, k}$ is less than $R$ and zero for vice versa. After calculating $\xi$ for each sub-cell, an average of all the sub-cell $\xi$ values will give the value of $\eta$ for the whole cell (see Fig. 2(a)). Not employing the sub-grid method in the vicinity of the solid sphere boundary will lead to a coarse body profile as shown in Fig. 3(a). The sub-grid method will produce a finer outline closer to the actual spherical shape as displayed in Fig. 3(b), without the need to modify the grid size of the computational domain.

Once the VOS is obtained, we can proceed to solve the governing equations. Firstly, the governing equations are discretized (eq. (2)) using the quadratic upstream interpolation for convective kinematics (QUICK) scheme proposed by Leonard (1979). A staggered grid is used in the present study. For the temporal term, the Adams-Bashforth scheme is applied. Using the projection method, the first intermediate velocity $\left(\mathbf{u}^{*}\right)$ is determined without the pressure gradient and virtual force term as follows

$\mathbf{u}^{*}=\mathbf{u}^{m}+\Delta t\left(\frac{1}{\operatorname{Re}} \cdot \nabla^{2} \mathbf{u}-\nabla \mathbf{u u}\right)^{m}$.

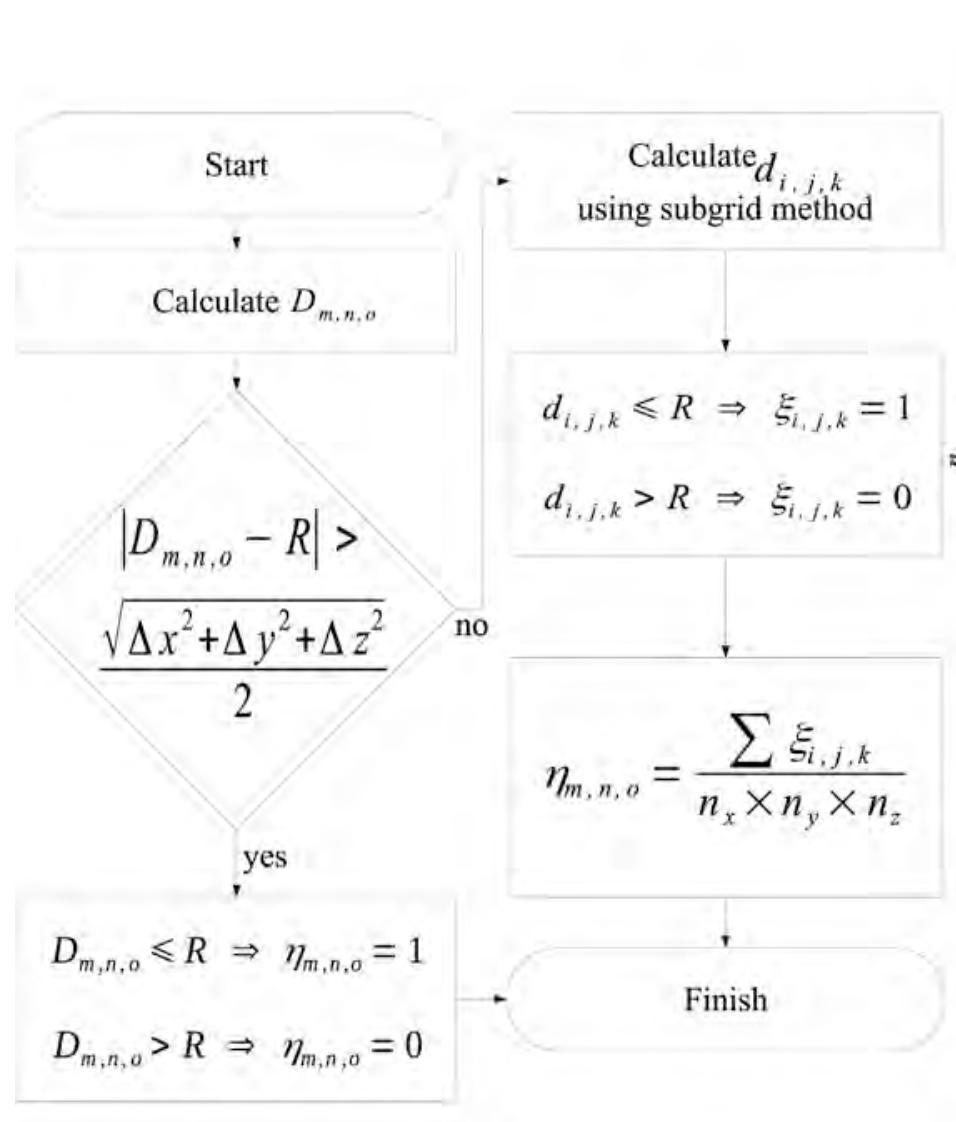

(a)

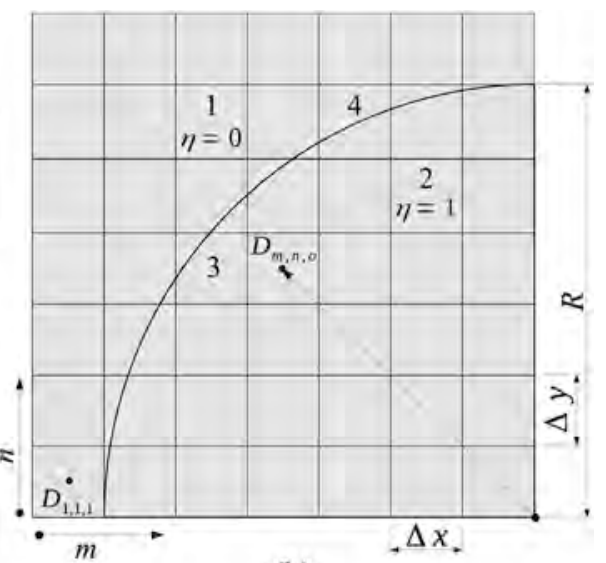

(b)

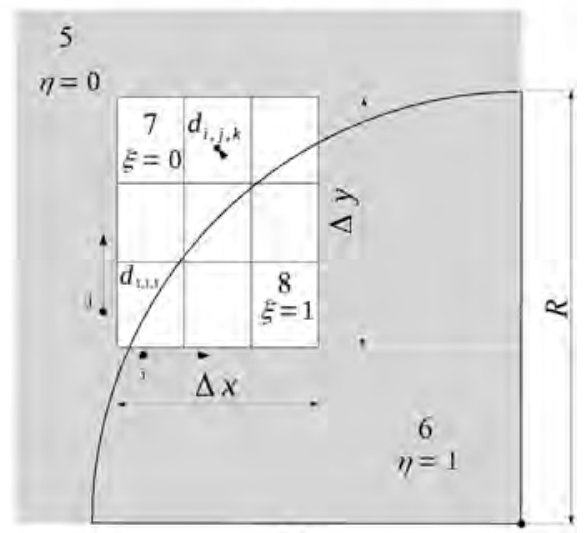

(c)

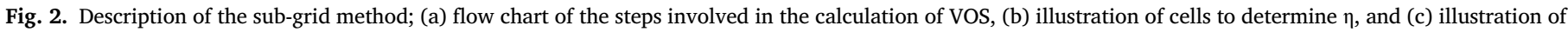
sub-cells to determine $\xi$. 


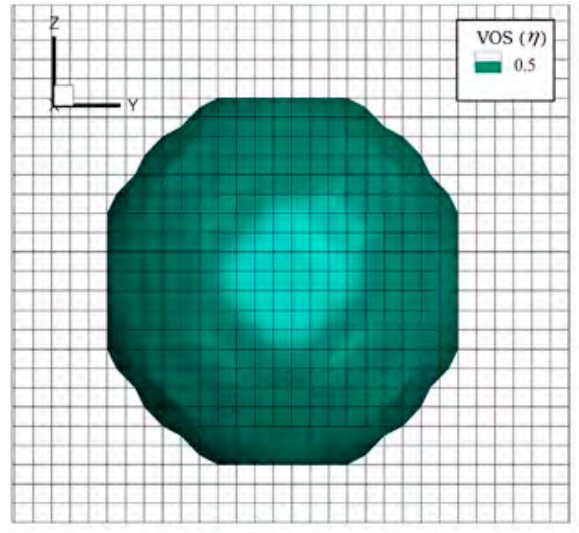

(a)

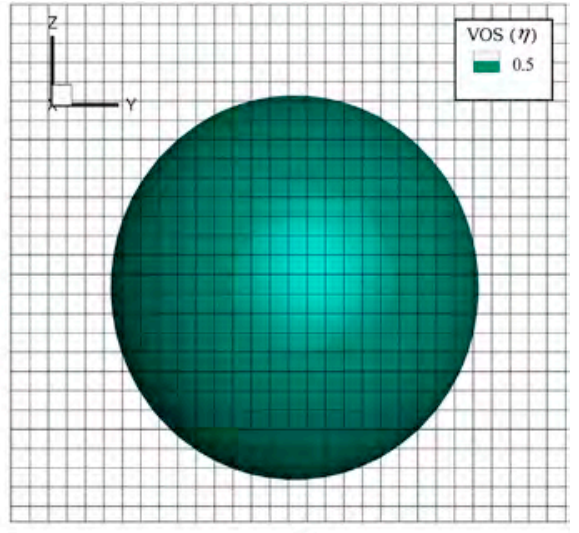

(b)

Fig. 3. Comparison of sphere profile (a) without sub-grid method and (b) with sub-grid method.

The next step involves solving the pressure Poisson equation using the continuity equation (eq. (1)) to find the second intermediate velocity $\left(\mathbf{u}^{* *}\right)$,

$\mathbf{u}^{* *}=\mathbf{u}^{*}-\nabla p^{m+\frac{1}{2}} \Delta t$.

The current study makes use of Successive Over-Relaxation (SOR) method to solve the pressure Poisson equation. Once the second intermediate velocity $\left(\mathbf{u}^{* *}\right)$ has been determined, the final corrected velocity is calculated by considering the virtual force $\left(\mathbf{f}^{*}\right)$ to account for VOS,

$\mathbf{f}^{*}=\eta \frac{\mathbf{u}_{s}^{m+1}-\mathbf{u}^{* *}}{\Delta t}$,

and

$\mathbf{u}^{m+1}=\eta \mathbf{u}_{s}^{m+1}+(1-\eta) \mathbf{u}^{* *}$,

where $\mathbf{u}_{s}^{m+1}$ represents solid motion velocity. The total force $(F)$ that occurs on the rigid-body at a certain time is given by

$F=-\iiint_{\Omega_{s}} \eta \mathbf{f}^{*} d V$

\subsection{Equations of motion of the rigid-body}

The rigid-body motion needs to be analyzed to determine the velocity of sphere motion and the sphere position at every time step. As the sphere is restrained in two directions, its motion is only in the transverse direction. Non-dimensionalizing the governing equation of rigid-body motion (eq. (3)), the following equation can be obtained for the $y$ -

Table 1

Dimensionless variables for rigid-body motion equations.

\begin{tabular}{lll}
\hline Time & $t^{*}$ & $\frac{t U_{\infty}}{D}$ \\
Displacement & $Y$ & $\frac{d_{y}}{D}$ \\
Mass ratio & $m^{*}$ & $\frac{m_{s}}{\frac{1}{6} \pi \rho_{f} D^{3}}$ \\
Reduced velocity & $U_{R}^{*}$ & $\frac{U_{\infty}}{f_{n} D}$ \\
Structure damping ratio & $\zeta$ & $\frac{c}{2 \sqrt{m_{s} k}}$ \\
Drag force coefficient & $C_{D}$ & $\frac{F_{x}}{2} \rho_{f} U_{\infty}^{2}\left(\pi D^{2} / 4\right)$ \\
Lift force coefficient & $C_{L}$ & $\frac{F_{y}}{1}$ \\
& & $\frac{1}{2} \rho_{f} U_{\infty}^{2}\left(\pi D^{2} / 4\right)$ \\
\hline
\end{tabular}

direction.

$\ddot{Y}+\frac{4 \pi \zeta}{U_{R}^{*}} \dot{Y}+\left(\frac{2 \pi}{U_{R}^{*}}\right)^{2} Y=\frac{3 C_{L}\left(t^{*}\right)}{4 m^{*}}$

where $\ddot{Y}, \dot{Y}$ and $Y$ are the normalized acceleration, velocity and displacement of the sphere's center in the transverse direction. Table 1 is a complete description of the dimensionless variables used in eq. (9). The rigid-body motion is described by a second-derivative equation. In the present work, we used a fourth-order Runge-Kutta method to solve this equation numerically.

$D$ is the dimensionless characteristic length of the rigid-body, which, in this case, is the diameter of the transversely vibrating sphere. The variable $d_{y}$ represents the displacement of the sphere's center in the $y$ direction. Mass ratio is the ratio between rigid-body mass, $m_{s}$, and the mass of fluid with the same spherical volume. The mass of fluid can be calculated using $\frac{1}{6} \pi \rho_{f} D^{3}$ where $\rho_{f}$ is the density of fluid. Reduced velocity, $U_{R}^{*}$, is ratio of the force exerted by fluid on the structure and the elastic restoring force of the structure. We know from the work of Feng (1968), a regime of normalized velocity $\left(U_{R}^{*}\right)$ takes place when the oscillation frequency of the sphere coincides with the frequency vortices formed behind it. In real-world applications, the value of $U_{R}^{*}$ is affected by the structure of the rigid-body.

The VIV phenomena can be investigated by using average drag coefficient $\left(\overline{C_{D}}\right)$, root mean square of lift coefficient $\left(C_{L, \text { rms }}\right)$, frequency ratio $\left(f^{*}\right)$, amplitude ratio in the transverse direction $\left(A_{y}^{*}\right)$ and energy efficiency $(E)$, as shown in Table 2. $f_{V}$ and $f_{N}$ are the static body vortex shedding frequency and the natural frequency of the system, respectively. A value of $f^{*}$ close to 1 generally indicates that any rigid-body vibrations occurring are caused by the vortex shedding behind the rigid-body, which is a sphere in this case. $P_{F-B}$ is the energy involved in the VIV phenomena whereas $P_{F}$ is the total energy of the fluid flow over the rigid-body.

Table 2

Evaluation parameters of VIV phenomena.

\begin{tabular}{lll}
\hline Amplitude ratio (y-direction) & $A_{y}^{*}$ & $\sqrt{2}\left(\frac{Y_{\text {rms }}}{D}\right)$ \\
Frequency ratio & $f^{*}$ & $\frac{f_{V}}{f_{N}}$ \\
Energy efficiency & $E$ & $\frac{P_{F-B}}{P_{F}}$ \\
& $P_{F-B}$ & $\frac{1}{T} \int_{0}^{T} F_{y} \ddot{Y} d t$ \\
& $P_{F}$ & $\frac{1}{8} \rho \pi d^{2} U_{\infty}^{3}$ \\
\hline
\end{tabular}


Table 3

The boundary conditions and initial conditions.

\begin{tabular}{lllll}
\hline Wall & $u$ & $v$ & $w$ & $p$ \\
\hline North & 1.0 & 0.0 & 0.0 & 0.0 \\
South & 1.0 & 0.0 & 0.0 & 0.0 \\
East & $d u / d x=0.0$ & $d v / d x=0.0$ & $d w / d x=0.0$ & $d p / d n=0.0$ \\
West & 1.0 & 0.0 & 0.0 & 0.0 \\
Front & 1.0 & 0.0 & 0.0 & 0.0 \\
Back & 1.0 & 0.0 & 0.0 & 0.0 \\
Initial condition & 1.0 & 0.0 & 0.0 & 0.0 \\
\hline
\end{tabular}

Table 4

Grid independence test results for the flow past a stationary sphere using $\operatorname{Re}=$ 50.

\begin{tabular}{lllll}
\hline & Grid A & Grid B & Grid C & Grid D \\
\hline Number of grids & $1,051,875$ & $1,423,325$ & $1,636,200$ & $2,120,000$ \\
$\Delta t$ & $1.0 \times 10^{-4}$ & $7.5 \times 10^{-5}$ & $6.0 \times 10^{-5}$ & $5.0 \times 10^{-5}$ \\
Smallest $\Delta x_{i}$ & 0.066 & 0.05 & 0.04 & 0.033 \\
Final time step & $2.7 \times 10^{5}$ & $3.44 \times 10^{5}$ & $5.87 \times 10^{5}$ & $7.12 \times 10^{5}$ \\
$C_{D}$ & 1.70 & 1.62 & 1.563 & 1.561 \\
\hline
\end{tabular}

Table 5

Comparison of drag coefficient and re-circulation length at $\mathrm{Re}=50$.

\begin{tabular}{lll}
\hline Authors & $\overline{C_{D}}$ & $x_{r}$ \\
\hline Taneda (1956) & - & 0.445 \\
Johnson and Patel (1999) & 1.558 & 0.413 \\
Roos and Willmarth (1971) & 1.558 & - \\
Tomboulides et al. (1993) & - & 0.405 \\
Present study & 1.563 & 0.433 \\
\hline
\end{tabular}

\section{Results and discussion}

Out of the three cases, the first case of flow past a sphere was investigated to validate the in-house developed solver. In the second case, numerical simulations were conducted on the phenomenon of transverse VIV of a sphere for comparison with previous studies. For the third case, a small stationary sphere is placed upstream of the larger vibrating sphere and the effects of the small sphere on VIV of larger sphere were studied by varying the diameter of the small sphere $\left(d_{\text {small }}\right)$ and the gap between the two $\left(x_{\text {gap }}\right)$. All simulations were started with the sphere at rest position. This means that the reduced velocity or any of the parameters were not changed during the simulation and new simulations were run for each case. The parameters used to analyze the VIV phenomena are drag coefficient, lift coefficient, amplitude ratio, frequency ratio, efficiency energy and velocity profile behind a small stationary sphere. Before a discussion of the VIV phenomena for the second and third cases, the validation of the solver and numerical sensitivity studies are discussed.

\subsection{Validation and numerical sensitivity studies}

Validation studies for the solver ensure that the in-house developed solver can produce correct results, while numerical sensitivity studies aim to obtain the optimal parameters for the investigation, especially the values of $\Delta x$ for the grid and the time step $\Delta t$. The cases of flow past a stationary sphere as well as the vibrating sphere are considered. First, validation is conducted for flow past a sphere at $R e=50$. This case aims to test the convergence of the solver and prove the suitability of the coded DFIB method for the 3D case. Earlier studies have shown that flow past a sphere is steady only up to $\mathrm{Re}=210$ approximately (Tomboulides et al., 1993). Therefore, a lesser value of $\operatorname{Re}=50$ was chosen for validation of the steady case for direct comparison with published literature.

The schematic of the computational domain for the case of flow past a sphere at $\mathrm{Re}=50$ is similar to that shown in Fig. 1 (without the smaller sphere and VIV) and the boundary conditions are presented in Table 3. A grid independence test is carried out to find the optimal grid for the investigation. Finer grids will provide more accurate results but take a longer computational time. At this stage, four variations of the number of grids are tested, namely the grids A, B, C and D as presented in Table 4. Grid independence test is conducted by a comparison of the results after grid refinement, while considering the CFL number. This also means that the finer the grid the smaller $\Delta t$ must be. These tests were performed for non-uniform grids selected optimally for the problem under consideration by decreasing the grid size around the sphere.

The results of the grid independence test are shown in Table 4. Using the value of drag coefficient to analyze the independence of the grid, Grid $\mathrm{C}$ is found to be optimal after comparison with published literature. The re-circulation length $\left(x_{r}\right)$ and drag coefficient $\left(C_{D}\right)$ are compared with previous studies and presented in Table 5 for the vorticity contours of Fig. 4. In general, the results shown in this study are quite similar to previous studies.

The validation of the developed code for VIV phenomena is based on the parameters used by Behara et al. (2011). The parameters and boundary conditions used for the transversely vibrating motion of the sphere at $\mathrm{Re}=300$ are presented in Table 6 and Table 3, respectively. The computational domain is similar to that shown in Fig. 1 (without the smaller sphere). Similar to the stationary sphere case, the validation in the case of VIV phenomenon also begins with the grid independence test to obtain optimal results before comparison with published studies. The results of this grid independence test are presented in Table 7. Grid L was eventually selected (shown in Fig. 5) as further refinement of the grid did not lead to significant improvement in results. With parallelization using OpenMP, this grid took almost 3 days to solve using the hardware described in section 1.3. There are only a few studies that have been done for VIV phenomenon at $\mathrm{Re}=300$. For the present work, the results are compared with those of Behara et al. (2011) and Rajamuni et al. (2018b). The validation was conducted at a reduced velocity of 6.0 because it is observed from both the previous studies that the maximum amplitude ratio occurs at $U_{R}^{*}=6.0$.

Table 8 presents a comparison of drag coefficient $\left(C_{D}\right)$, lift coefficient $\left(C_{L, \text { rms }}\right)$, amplitude ratio in the transverse direction $\left(A_{y}^{*}\right)$ and frequency ratio $\left(f^{*}\right)$ with previous studies. Based on this comparison, it can be seen that the average of drag coefficient, amplitude ratio and frequency ratio are close to previous results. However, the root mean square value of the lift coefficient $\left(C_{L}^{r m s}\right)$ is different from the results of Behara et al. (2011) but quite close to those of Rajamuni et al. (2018b).

\subsection{Transverse vibration of a single sphere}

The same parameters, computational domain and boundary
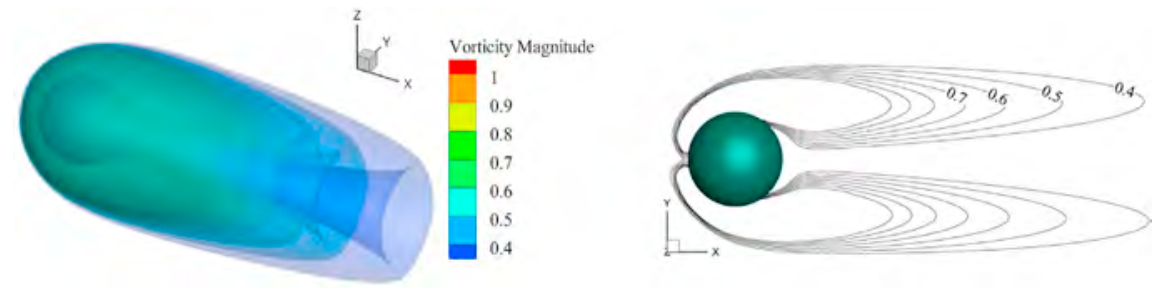

Fig. 4. Vorticity contours, $\mathrm{Re}=50$. 
Table 6

Transverse vibrating motion parameters.

\begin{tabular}{llll}
\hline Mass ratio $\left(m^{*}\right)$ & Re & Reduced velocity $\left(U_{R}^{*}\right)$ & $\zeta$ \\
\hline 3.82 & 300 & 6.0 & 0.0 \\
\hline
\end{tabular}

Table 7

Grid independence test results for the transverse vibration of the sphere using similar parameter values as Behara et al. (2011).

\begin{tabular}{llll}
\hline & Grid K & Grid L & Grid M \\
\hline Number of grids & 193,600 & 865,208 & $1,820,216$ \\
$\Delta t$ & $1.6 \times 10^{-3}$ & $8.0 \times 10^{-4}$ & $6.5 \times 10^{-4}$ \\
Smallest $\Delta x_{i}$ & 0.1 & 0.05 & 0.04 \\
Final time step & $2.0 \times 10^{5}$ & $3.75 \times 10^{5}$ & $5.00 \times 10^{5}$ \\
$\overline{C_{D}}$ & 0.8101 & 0.78769 & 0.7834 \\
$C_{L, \text { rms }}$ & 0.2313 & 0.31597 & 0.3118 \\
$f^{*}$ & 0.8152 & 0.84397 & 0.8339 \\
$A_{y}^{*}$ & 0.2522 & 0.31597 & 0.3221 \\
\hline
\end{tabular}

conditions are used as those for validation. An analysis of the variation of reduced velocity is also conducted. The parameters used in this case are in accordance with the work of Behara et al. (2011) (see Table 6) with variation of reduced velocity from 3.5 to 9.0. Similar parameters were used by Rajamuni et al. (2018b).

\subsubsection{Sphere responses}

In this case, the rigid-body in the problem is a single sphere that is free to oscillate transverse to the flow in $y$-direction. The case schematic, VIV parameters and boundary conditions are exactly the same as those during validation of VIV. However, a range of values for reduced velocity have been used. Validation case is conducted for $U_{R}^{*}=6.0$, while further analysis requires the variation of $U_{R}^{*}$ from 3.5 to 9.0. This allows us to examine the effects of reduced velocity on the system and identify the lock-in regime. Fig. 6(a) shows the values of amplitude ratio with variations in reduced velocity. In addition, comparison between the present study and the results of previous studies, namely Behara et al. (2011), Behara and Sotiropoulos (2016) and Rajamuni et al. (2018b), can also be seen in Fig. 6(a).

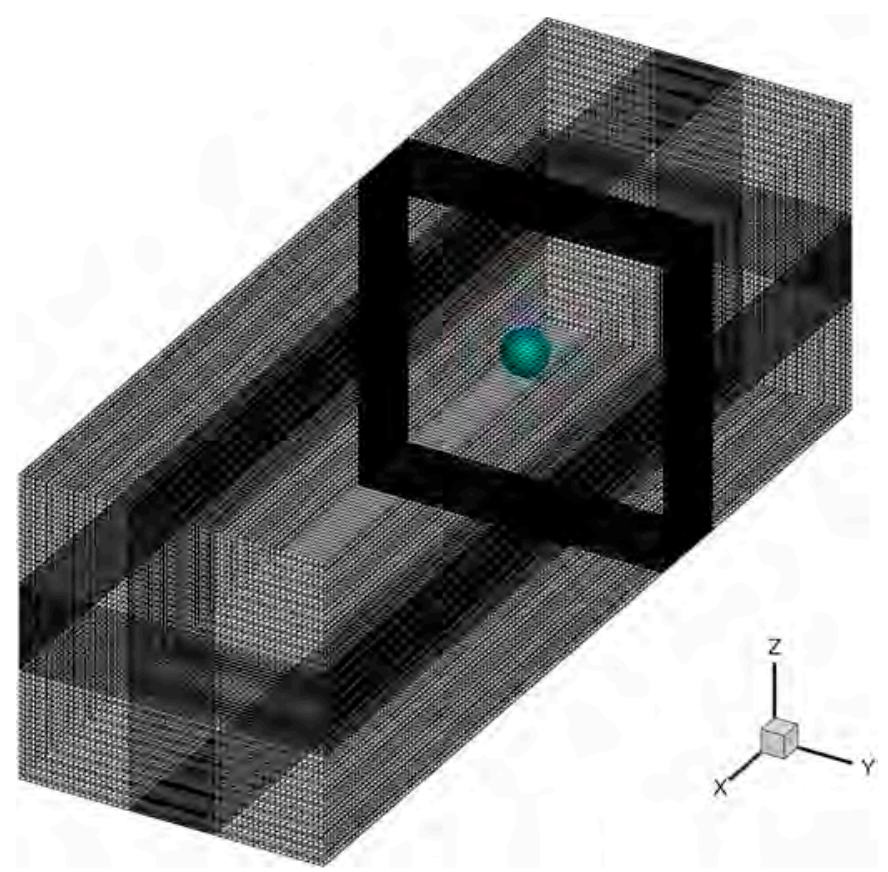

Fig. 5. Grid L tested during the grid independence analysis and adopted for the rest of the study.
Table 8

Comparison of drag coefficient, lift coefficient, amplitude ratio and frequency ratio for transverse vibration at $\mathrm{Re}=300$.

\begin{tabular}{lllll}
\hline Authors & $\overline{C_{D}}$ & $C_{L, \mathrm{rms}}$ & $A_{y}^{*}$ & $f^{*}$ \\
\hline Behara and Sotiropoulos (2016) & 0.752325 & 0.085821 & 0.330754 & 0.9397 \\
Rajamuni et al. (2018b) & 0.837287 & 0.2598 & 0.3620 & - \\
Present study & 0.78769 & 0.31597 & 0.31597 & 0.84397 \\
\hline
\end{tabular}

In their study, Behara et al. (2011) represent mass with the structural mass of solid, $m_{s}=2.0$, which is equivalent to a mass ratio of $m^{*}=3.82$. Meanwhile, Rajamuni uses the same approach in this regard as the present study. For the current investigation, the maximum amplitude ratio occurs at $U_{R}^{*}=5.6$ while the lock-in regime starts close to $U_{R}^{*}=5.0$. From Fig. 6(a), it can be seen that the maximum amplitude of vibration lies in the same range as previous studies. The beginning of the lock-in regime, characterized by high vibration amplitudes, is close to the work of Behara et al. (2011) and Behara and Sotiropoulos (2016). A possible reason for the difference in results between the present study and those of Rajamuni et al. (2018b) is blockage ratio. Rajamuni et al. (2018b) conducted their analysis for a very large computational domain with respect to the sphere, leading to a very small blockage ratio. However, most published numerical studies do not use such large computational domains. Considering the trend of frequency ratio in the present study, it is also similar to the results of Behara et al. (2011) as shown in Fig. 6(b). An increase in reduced velocity leads to an increase in the frequency ratio. Calculation of $f^{*}$ uses FFT analysis. After the onset of VIV, the frequency ratio ranges from 0.79 to 0.93 or close to 1 with no stream velocity in the $y$-direction, which shows that the oscillation of the sphere is caused by vortex shedding behind the sphere.

\subsubsection{Vortex shedding modes}

A discussion of the visualization of vortex shedding occurring due to the VIV phenomenon for a single sphere is now discussed. The visualization is achieved using the method of Q-criterion, developed by Hunt et al. (1988). The Q-criterion value adopted in the present work is 0.001 . As shown in Fig. 7, four values of reduced velocity have been used, i.e. 4.0, 5.0, 5.6 and 9.0. $U_{R}^{*}=4.0$ was selected to analyze the vortices before the onset of the lock-in regime and it is observed that the type of vortex shedding occurring at this stage is also in the hairpin shedding mode regime, as per the terminology used in earlier studies. The height of the vortex shedding mode (distance between the positive $y$-direction and negative $y$-direction of the vortex structures) is quite small because the vibrations that occur are also very small or almost non-existent.

As $U_{R}^{*}$ increases to 5.0 , the vibrations get progressively larger (see Fig. 7(b)) eventually leading to the lock-in regime. The hairpin vortex shedding mode is still in effect with larger vortex structures until reaching the peak amplitude point at $U_{R}^{*}=5.6$ (see Fig. 7(c)). This mode also occurred in previous studies conducted by Behara et al. (2011) and Rajamuni et al. (2018b). After reaching the peak amplitude ratio $\left(A_{y}^{*}\right)$, the vortex shedding mode transforms from hairpin mode to a wake that is quite similar to that of a fixed sphere. This can be seen clearly in Fig. 7(d). The transition of vortex shedding modes also occurred in previous studies (Govardhan and Williamson, 2005; Behara et al., 2011; Rajamuni et al., 2018b). Since the sphere in the present study is restrained to move only in the transverse direction, a true spiral mode of vortex-shedding is not possible.

The time histories of normalized transverse amplitudes are shown in Fig. 8. At first the rigid-body was in a stationary position but with the passage of time due to the vortex shedding behind the it, it began to vibrate in the transverse direction. The vibration of the rigid-body continues to increase until at a certain point its amplitude stops increasing further and the movement of the rigid-body becomes identically repetitive. An that time the lock-in regime is said to have begun. In addition, 


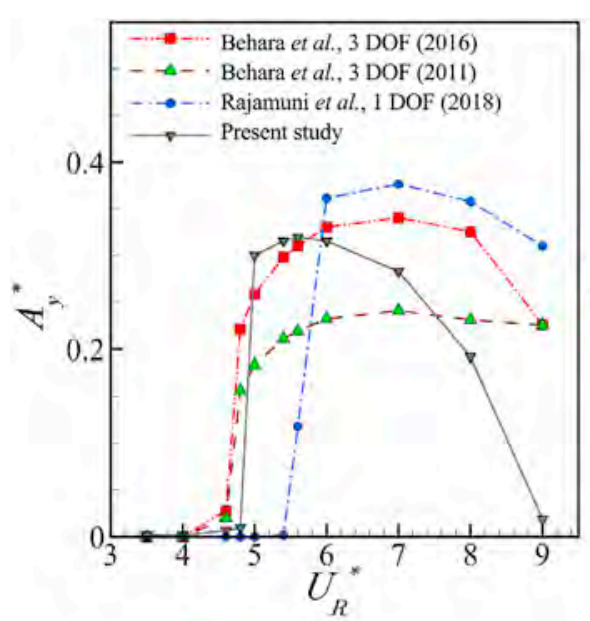

(a)

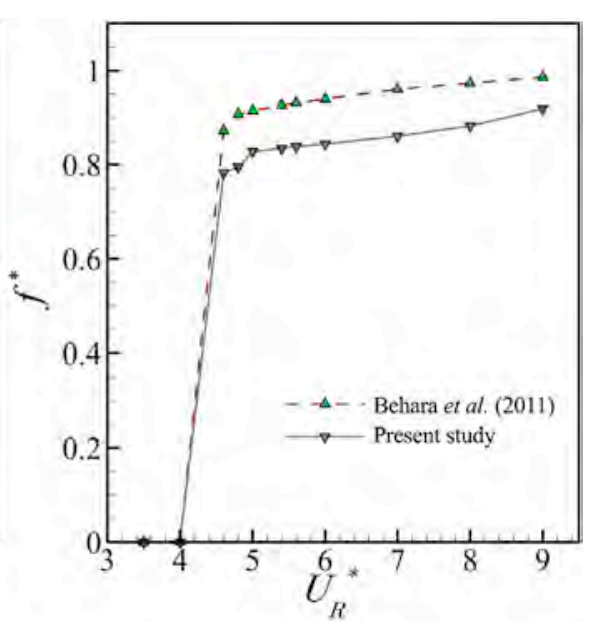

(b)

Fig. 6. Variation of (a) amplitude ratio $\left(A_{y}^{*}\right)$ and (b) frequency ratio $\left(f^{*}\right)$ in the transverse direction against reduced velocity, $U_{R}^{*}$.

the occurrence of the lock-in regimes is also influenced by the value of $U_{R}^{*}$ as can be seen in Fig. 8(a), as at $U_{R}^{*}=4.0$ the vibration of the rigid-body is quite small and it can be concluded that the lock-in regime does not occur. The case of $U_{R}^{*}=5.6$ in Fig. 8(c), where the lock-in regime began at $t^{*}=169.5$, requires a shorter time to achieve lock-in than the case of $U_{R}^{*}=5.0$ shown in Fig. 8(c), where lock-in onset occurred at $t^{*}=225.0$. As stated earlier, the peak point of amplitude ratio occurs at $U_{R}^{*}=5.6$. In general, with increase in $U_{R}^{*}$ after the maximum amplitude ratio, the amplitude ratio decreases gradually and the lock-in regime occurs at earlier times. For the $U_{R}^{*}=9.0$ case in Fig. 8(d), the lock-in regime occurs earlier at $t^{*}=44.72$.

\subsubsection{Force analyses}

Fig. 9(a) shows the comparison of average drag force in the present study with previous studies. The results of the present study generally appear to be in close agreement with existing literature. The trend of the graph with increase in $U_{R}^{*}$ is also exactly the same. Before the onset of the lock-in regime, the value of the average drag coefficient $\overline{C_{D}}$ is stable, as in previous studies. The rise in $\overline{C_{D}}$ values starts at the onset of the lock-in regime and reaches a maximum around the point when the sphere achieves its maximum vibration before gradually declining until the end of the lock-in regime. Unlike the $\overline{C_{D}}$ graph, the maximum for $C_{L, \text { rms }}$ shown in
Fig. 9(b) occurs at the onset of the lock-in regime. Before lock-in regime, the values of $C_{L, \mathrm{rms}}$ are almost zero and after the peak value, $C_{L, \mathrm{rms}}$ decreases gradually until the end of the lock-in regime. In comparison to previously published literature (Behara et al., 2011; Behara and Sotiropoulos, 2016; Rajamuni et al., 2018b), the maximum value of $C_{L, \mathrm{rms}}$ in the present study is higher than others and consequently, the rate of decline is also steeper. However, the overall trend of the curve is mostly the same.

The present results for the single sphere are in good agreement with the published literature. Apart from validating the current in-house developed code, this also confirms the suitability of the current model and use of direct-forcing immersed boundary method to study 3D VIV phenomenon for a sphere.

\subsection{Transverse vibration of a sphere downstream of a smaller fixed sphere}

Having examined the results of simulations for a single sphere, now the effects of adding a smaller fixed sphere before the larger sphere are discussed. Parameters and boundary conditions (see Tables 3 and 6) in this case are exactly same with those of the single sphere case. The difference is the addition of a smaller fixed sphere in front of the vibrating rigid-body. Computational domain for this case can be seen in Fig. 1. The investigation also analyzes the effects of variation of gap between the

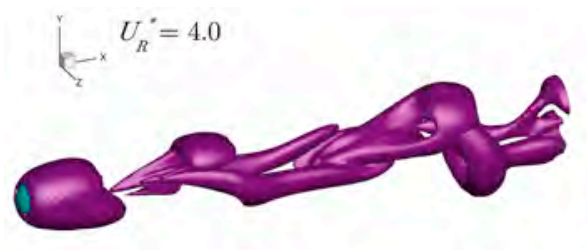

(a)

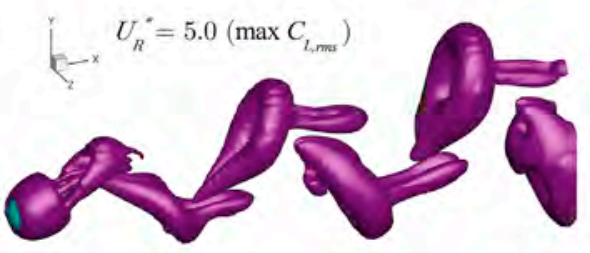

(b)

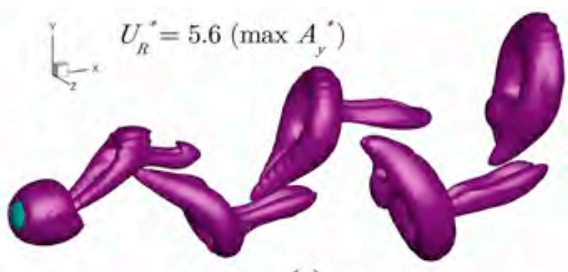

(c)

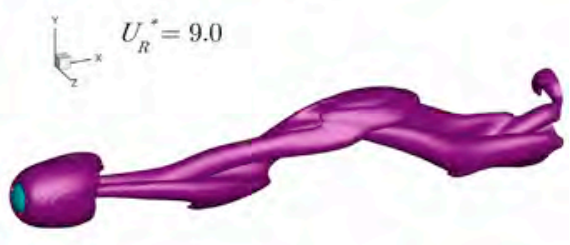

(d)

Fig. 7. Vortex shedding of a sphere visualized by Q-criterion (Hunt et al., 1988) with variation of reduced velocity; (a) $U_{R}^{*}=4.0$, (b) $U_{R}^{*}=5.0$, (c) $U_{R}^{*}=5.6$, (d) 


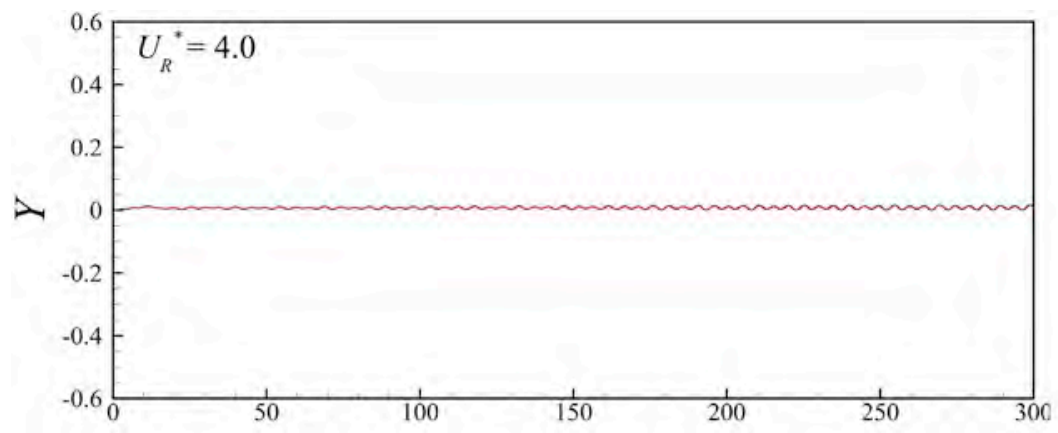

(a)

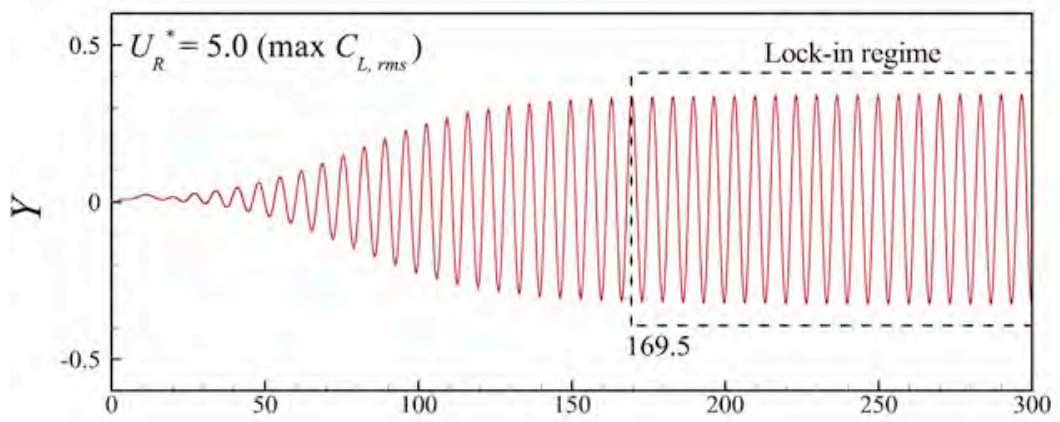

(b)

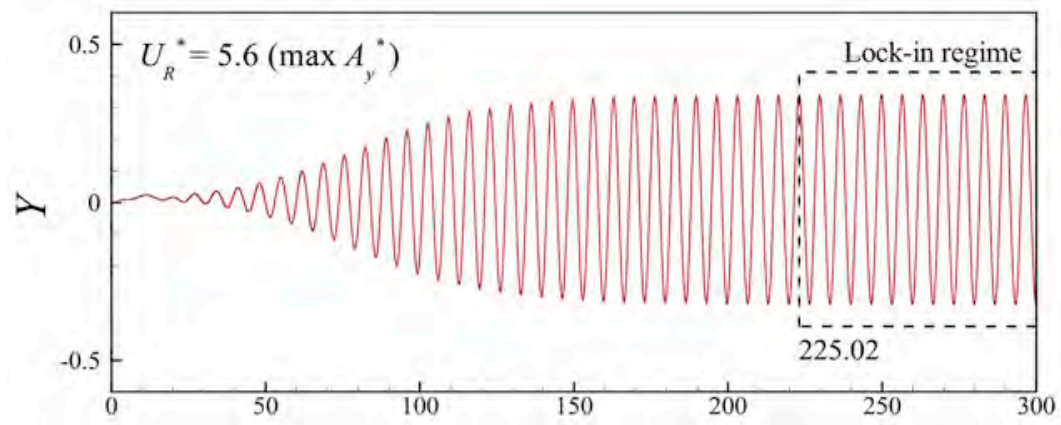

(c)

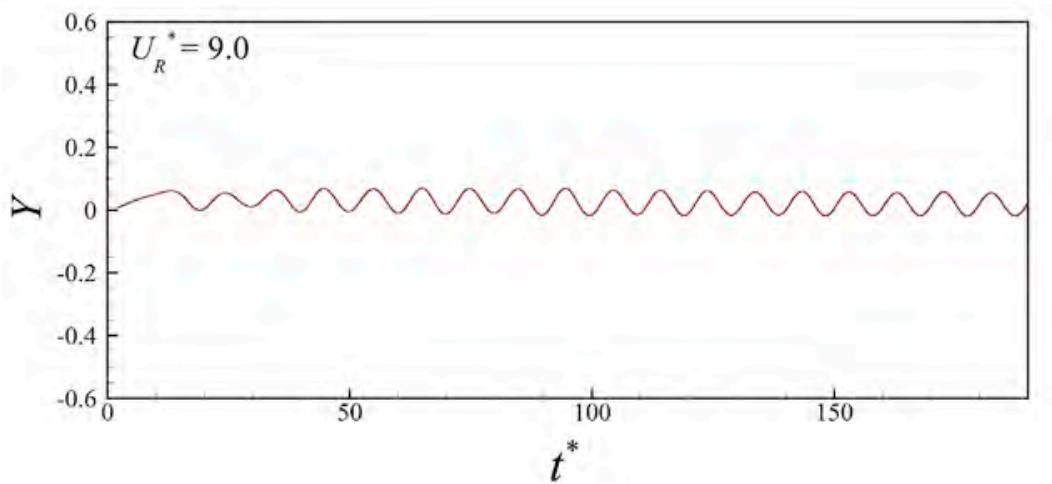

(d)

Fig. 8. Time histories of the normalized transverse displacement for different values of reduced velocity; (a) $U_{R}^{*}=4.0$, (b) $U_{R}^{*}=5.0$, (c) $U_{R}^{*}=5.6$, (d) $U_{R}^{*}=9.0$.

spheres $\left(x_{\text {gap }}\right)$ and the diameter of the smaller sphere $\left(d_{\text {small }}\right)$. The investigated values of $x_{\text {gap }}$ are $0.1 D, 0.3 D, 0.5 D$ and $0.7 D$, while $d_{\text {small }}$ values are $0.1 D, 0.2 D, 0.3 D$ and $0.4 D$, where $D$ is the diameter of the larger sphere. The analysis of this case also includes sphere responses, vortex shedding modes, time histories of the normalized transverse displacement as well as the hydrodynamic force measurements in the transverse and inline directions.

\subsubsection{Sphere responses}

The parameters used to discuss sphere response are amplitude ratio $\left(A_{y}^{*}\right)$ and frequency ratio $\left(f^{*}\right)$. As the sphere is restrained in other directions, the amplitude ratio measured in this study was is only for the transverse $y$-direction (see Table 2). Meanwhile, the frequency ratio is obtained from FFT analysis. Fig. 10(a) shows the variation of amplitude ratio with different smaller sphere diameters while the gap between the spheres is fixed at $x_{\text {gap }}=0.1 D$. The results show that the addition of the smaller sphere upstream of the rigid-body can lead to larger amplitude ratios of the rigid-body. Moreover, the greater the value of $d_{\text {small }}$, the larger is the amplitude ratio. Hence, it can be concluded that the VIV of the sphere becomes stronger due to the upstream smaller sphere. This seems to be due to the effect of wake-induced vibrations (WIV) described in section 1.1. Earlier research (Alam et al., 2003; Mysa et al., 2016) explains that an elastic object undergoes WIV when it is placed downstream within the wake of another object. The vibrating behavior of the downstream object among the two tandem objects increases rapidly in comparison to the vibration of an isolated object undergoing resonant VIV (Assi, 2014). Assi et al. (2010) described the interference of the flow between two identical objects placed in front of one another, even when 


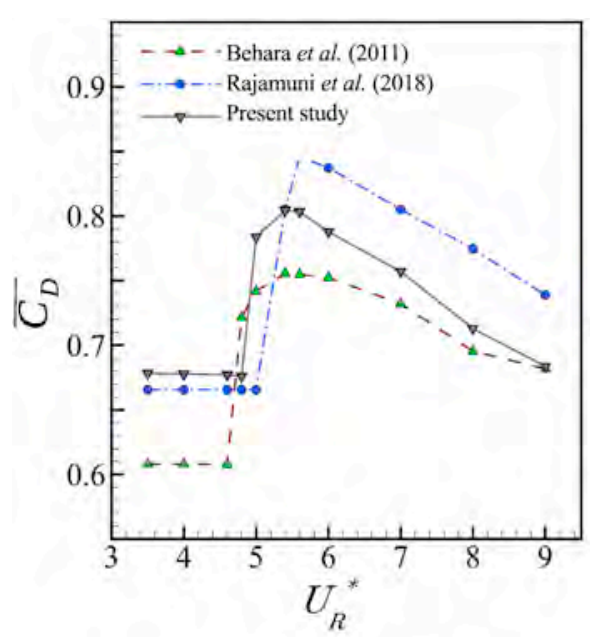

(a)

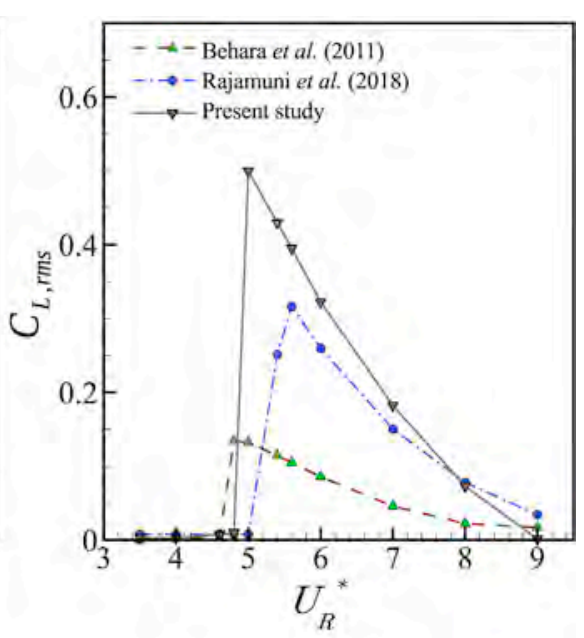

(b)

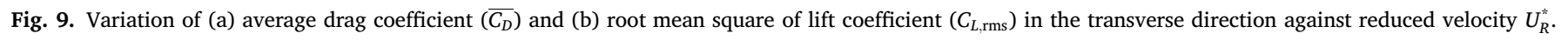

the gap between them is increased to up to 20 diameters. They have described how the vortices of the upstream body can cause high-amplitude vibrations of the downstream object. In the present study, the same effect appears to exist for non-identical objects, specifically when the upstream object is a sphere of a much smaller diameter and remains fixed. In fact, the peak amplitude ratio of the largest upstream sphere considered having size $d_{\text {small }}=0.4 D$ is twice the amplitude ratios of the case with no upstream sphere.

The overall trend for all values of $d_{\text {small }}$ in Fig. 10(a) is largely the same, even though the amplitude ratios for the largest two $d_{\text {small }}$ values do not drop to zero for the investigated reduced velocities. This also sheds light on another aspect of the VIV behavior. The lock-in regime onsets earlier as the size of $d_{\text {small }}$ is increased and remains in effect for a greater range of reduced velocities, for $d_{\text {small }} \geq 0.2 D$. Therefore, the introduction and increase in the size of the upstream smaller sphere enhances the amplitude ratio of VIV as well as the range of the lock-in regime with respect to reduced velocity.

From Fig. 10(b), it can be deduced that the VIV frequency ratio is largely unaffected by the introduction of the smaller sphere upstream with only slight changes in it for the different $d_{\text {small }}$ values. Except for the largest value of $d_{\text {small }}=0.4 D$, the frequency ratios for all other cases with the smaller sphere coincide in the initial part of the curve. However, focusing on the trend with increasing reduced velocity as shown in the inset figure, it is observed that smaller diameters of the upstream sphere lead to larger frequency ratios for the vibrating sphere and the highest values are attained for the case without the smaller sphere. Generally, the values of $f^{*}$ are less than one but the values of frequency ratio tend to be lower at larger $d_{\text {small }}$, perhaps because in these cases, the vibrations produced by vortex shedding behind the larger fixed sphere are unrelated to the enhanced WIV phenomenon caused by the small sphere.

The variable of $x_{\text {gap }}$, the distance between the spheres, also affects the magnitude of the amplitude ratio, as observed in Fig. 11. As the gap between the spheres increases, the impact of WIV phenomenon from the upstream smaller fixed sphere is reduced, leading to lower amplitude ratios of the vibrating sphere for a given reduced velocity, and the lowest is observed for the case without the sphere. The onset of the lock-in regime is only slightly affected by variation in $x_{\text {gap }}$. Both of these observations inform the view that the closer the smaller sphere is to the larger one, the greater influence it will have on the VIV of the larger sphere.

The effect of variation of $x_{\text {gap }}$ on the frequency ratio $\left(f^{*}\right)$ is not too significant as the curves for all cases with the smaller upstream sphere mostly coincide, as shown in Fig. 10(b). Looking at the inset figure though, a slight decrease in the frequency ratio is observed for greater

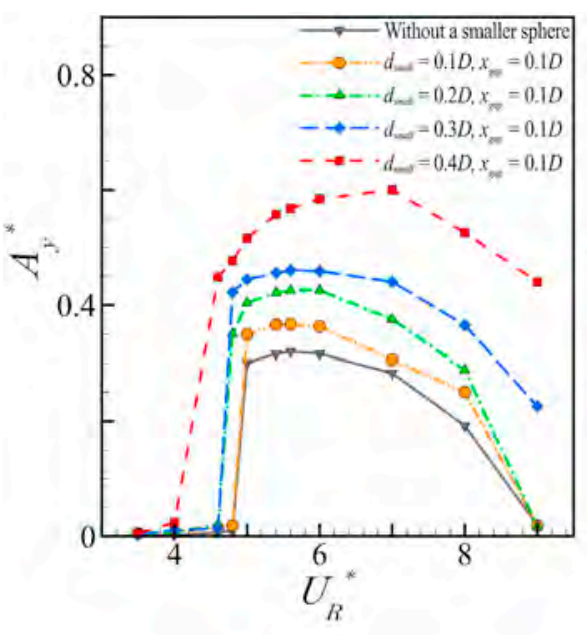

(a)

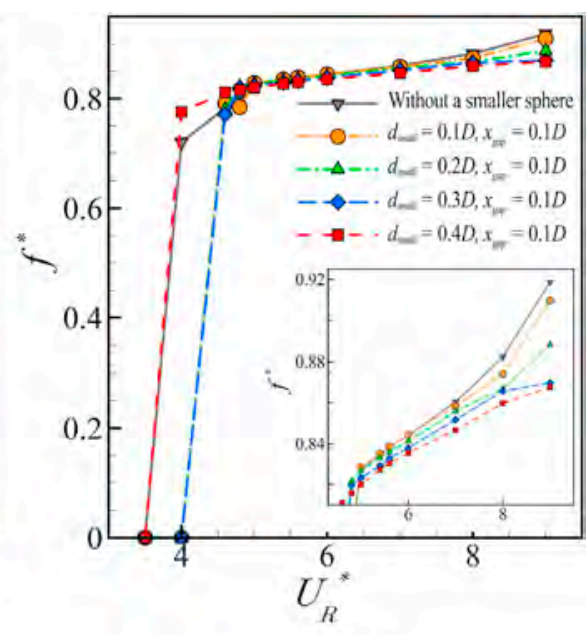

(b)

Fig. 10. Variation of (a) amplitude ratio $\left(A_{y}^{*}\right)$, (b) frequency ratio $\left(f^{*}\right)$, in the transverse direction for different small sphere diameters against reduced velocity $U_{R}^{*}$. 


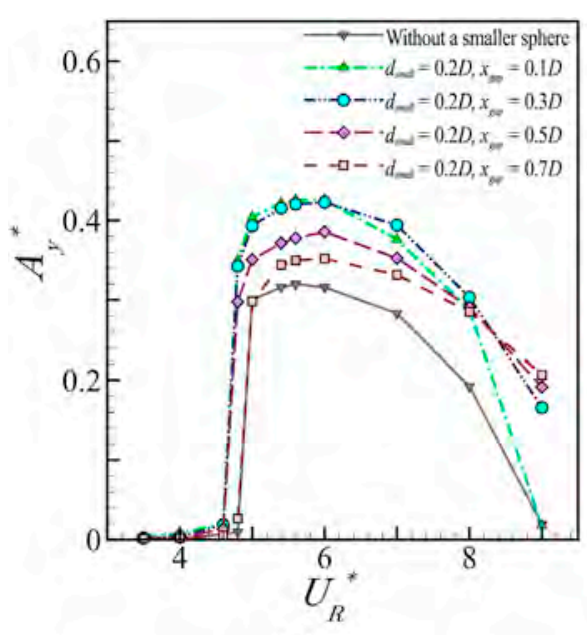

(a)

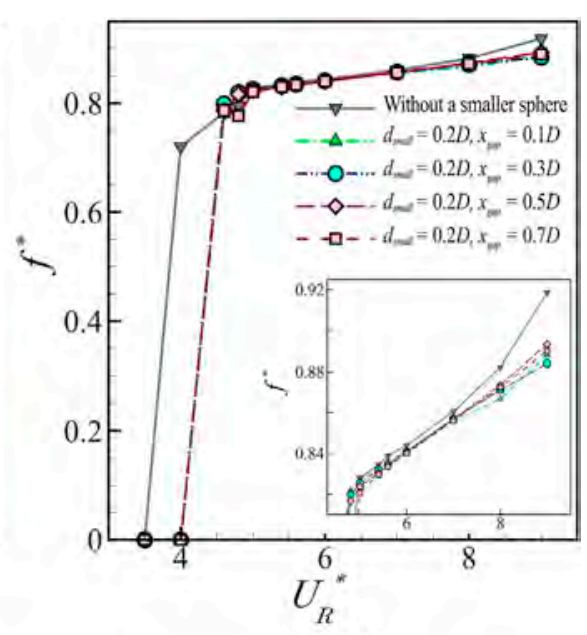

(b)

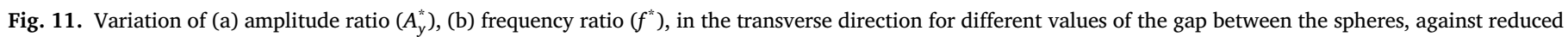
velocity $U_{R}^{*}$.

distances $x_{\text {gap }}$ between the spheres indicating the reduced impact of WIV phenomenon from the upstream small sphere, though no clear trend is discernible.

Summing up the amplitude response of the sphere, we can gather from the results that the amplitude increases rapidly with reduced velocity at the onset of synchronization in all the cases. It remains relatively high during the synchronization regime, before gradually decreasing as the synchronization/lock-in ends. Placing a small sphere upstream having any diameter $d_{\text {small }}$ increases the oscillating sphere's amplitude universally. However, the larger the $d_{\text {small }}$, the greater will be the amplitude. On the other hand, the amplitude tends to increase as the smaller fixed sphere is brought closer to the oscillating sphere ( $x_{\text {gap }}$ is decreased). However, the smallest value of $x_{\text {gap }}=0.1 D$ gives similar results to the case for $x_{\text {gap }}=0.3 D$ in the beginning of the synchronization regime while toward its end, the amplitude for the case of $x_{\text {gap }}=0.1 D$ falls rapidly. Such behavior indicates that there maybe an optimum distance between the spheres to enhance the amplitude but it needs to be investigated further.

\subsubsection{Vortex shedding modes}

Fig. 12 visualizes the vortex shedding using Q-criterion (Hunt et al., 1988) so that the evolution of vortex shedding modes can be assessed for different values of $x_{\text {gap }}$ and $d_{\text {small }}$. Three different combinations are selected for comparison. The first combination is for $x_{\text {gap }}=0.1 D$ and $d_{\text {small }}=0.2 D$. In the second combination, the distance between the spheres is the same constant but a larger diameter of the smaller sphere is

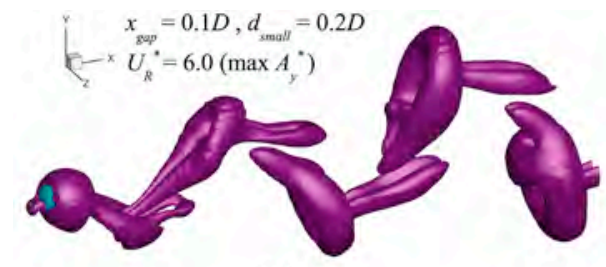

(a)

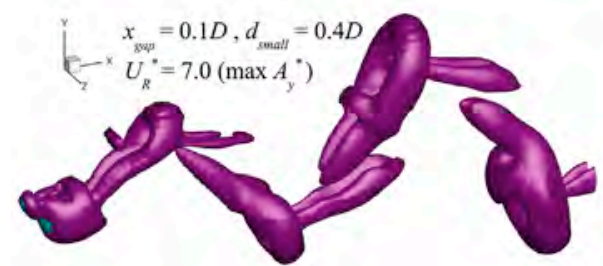

(b)

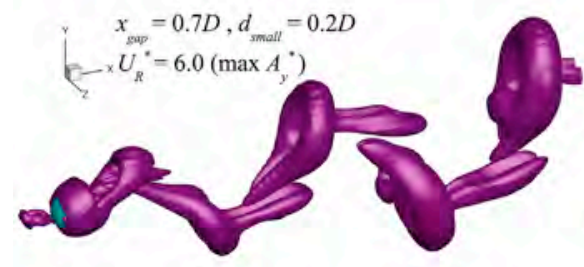

(c)

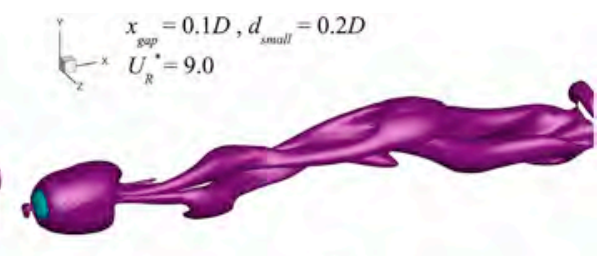

(d)

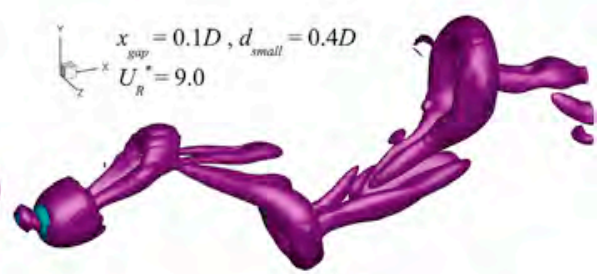

(e)

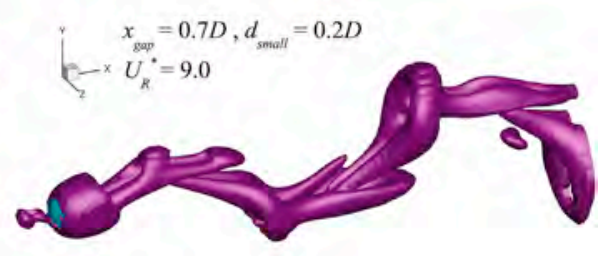

(f)

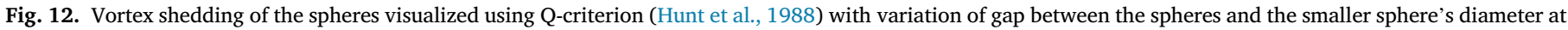
two reduced velocities: the reduced velocity at peak point amplitude ratio (maximum $A_{y}^{*}$ ) and after the peak amplitude ratio $\left(U_{R}^{*}=9.0\right.$ ). 
considered so that $x_{\text {gap }}=0.1 D$ and $d_{\text {small }}=0.4 D$. For the final combination, the diameter of the smaller sphere is the same as in the first combination but the gap between the spheres has been increased so that $x_{\text {gap }}=0.7 D$ and $d_{\text {small }}=0.2 D$. Visualization of the vortex shedding for each of these combinations is presented at two different reduced velocities; the reduced velocity at peak amplitude ratio and a reduced velocity after the peak amplitude ratio $\left(U_{R}^{*}=9.0\right)$, which is the maximum reduced velocity investigated in the present study.

The first combination $\left(x_{\text {gap }}=0.1 D\right.$ and $\left.d_{\text {small }}=0.2 D\right)$ is shown in Fig. 12(a) at the maximum amplitude ratio, where the type of vortex shedding is almost the same as that encountered behind a single sphere, namely the hairpin mode, which remained in effect for the single sphere from small vibration amplitudes at low reduced velocities to the maximum amplitude ratio at higher reduced velocities. It is reasonable to assume that even behind a small fixed sphere, the vortex shedding of the vibrating larger sphere would be in the hairpin mode at the smaller amplitudes too. After the lock-in regime, the vortex shedding for a single sphere (see Fig. 7) transformed from hairpin mode to a wake similar to that of a fixed sphere. The same phenomenon can be observed here in Fig. 12(d) where the vortex shedding mode transforms after the lock-in regime has ended (refer to Fig. 10). Therefore, the addition of a smaller fixed sphere upstream does not influence the type of vortex shedding modes.

For the second combination $\left(x_{\text {gap }}=0.1 D\right.$ and $d_{\text {small }}=0.4 D$ ) shown in Fig. 12(b), the vortex shedding patterns are quite similar to the hairpin mode of the first combination. Note that the reduced velocity considered here is higher at $U_{R}^{*}=7.0$ to coincide with the maximum vibration amplitude for this case. After the peak amplitude, at $U_{R}^{*}=9.0$, the vortex shedding height is reduced (Fig. 12(e)), though the mode is still hairpin because the vibrations are still sufficiently high. The hairpin mode disappeared in the first combination because of the end of the lock-in regime. Here, however, the lock-in regime still persists at $U_{R}^{*}=9.0$, as is also clear in Fig. 10.

The last combination ( $x_{\text {gap }}=0.7 D$ and $d_{\text {small }}=0.2 D$ ) has the same $d_{\text {small }}$ as the first combination but a greater distance $x_{\text {gap }}$ between the spheres. A similar vortex shedding pattern with hairpin mode can be seen in Fig. 12(c) at the peak amplitude ratio occurring at $U_{R}^{*}=6.0$. For $U_{R}^{*}=$ 9.0, the lower amplitude ratio means that the vortex shedding patterns are smaller in size but slightly more elongated at the higher reduced velocity. However, the amplitude ratio is still sufficiently high due to the lock-in effect (see Fig. 11), which is why the vortex shedding is still in hairpin mode. Nevertheless, the impact of the smaller fixed sphere on the vortex shedding patterns of the larger sphere appear to be minimal for all combinations of $x_{\text {gap }}$ and $d_{\text {small }}$ and hence, it can be reasonably assumed that within the ranges of these variables investigated in this study, the vortex shedding modes of a vibrating sphere behind a fixed smaller object will follow the same behavior as that of a single vibrating sphere but the height of the vortex structures would be larger as the presence of the smaller sphere upstream leads to larger vibration amplitudes $\left(A_{y}^{*}\right)$.

Fig. 13 presents a close-up of the spheres during vortex shedding for different values of $x_{\text {gap }}$ and $d_{\text {small }}$. The same combinations were selected as those discussed above for Fig. 12, at the respective reduced velocities corresponding to their maximum amplitude ratios. Clear vortices can be seen developing behind the smaller fixed sphere. For the first two combinations in Fig. 13(a) and (b), the gap between the spheres is the same. Although the developed vortices are small, they immediately impact the larger sphere and contribute their force to its oscillations.

Conversely, in the final combination having a larger gap, the vortex produced by the smaller sphere is not immediately received by the larger sphere, which could lead to some of its energy getting dissipated to the surrounding flow. Consequently, the decrease in vibration amplitudes with increasing gap between the spheres observed in Fig. 11 can be explained by some of the energy from the smaller sphere's vortices getting dissipated to the surrounding flow. On the other hand, the upstream fixed sphere having a larger diameter will produce greater vortex shedding and the energy contributed to the vibration of the larger sphere will also be greater, as can be deduced from the increasing vibration amplitudes with respect to $d_{\text {small }}$ shown in Fig. 10 .

Fig. 14 presents the time histories of the normalized transverse vibration of the vibrating sphere at the respective $U_{R}^{*}$ corresponding to peak amplitude ratios for different sizes of upstream small spheres. In addition to the increase in vibration amplitudes for larger sizes of upstream spheres, the time taken for the onset of the lock-in regime is also longer for larger $d_{\text {small values. }}$

Similarly, Fig. 15 displays the time histories of the normalized transverse vibration of the vibrating sphere at the respective $U_{R}^{*}$ corresponding to peak amplitude ratios for different distances between the spheres. Although a similar increase in the time required for onset of the lock-in regime can be observed with increasing values of $x_{\text {gap }}$, the time difference between the cases is quite small.

\subsubsection{Force analyses}

The parameters used for force analyses are average drag force coefficient $\left(\overline{C_{D}}\right)$ and root mean square of lift force coefficient $\left(C_{L, \text { rms }}\right)$. Both the parameters have been selected for this study due to their significant influence on VIV behavior of the sphere. Drag force coefficient represents the force generated by the fluid flow in the $x$-direction while lift force coefficient represents the forces occurring in the transverse $y$-direction due to oscillations of the rigid-body.

Fig. 16(a) shows the variation of average drag coefficient with respect to $U_{R}^{*}$ for four different $d_{\text {small }}$ values, as well as without the small sphere. Considering the curve for the case without a smaller sphere, the drag forces are observed to rise rapidly with the onset of the lock-in regime, which is consistent with published literature as discussed in section 3.2.3. All the cases with a small fixed sphere upstream generally have lower values of average drag coefficient in comparison to the case without the smaller sphere due to the effect of the vortex shedding behind the smaller sphere. Also, a general trend can be observed where the increase in the size of the fixed sphere upstream leads to an even lower drag force on the vibrating sphere. But the differences in drag forces between the case without the sphere and other cases with the sphere are much larger for the reduced velocities before the onset of the lock-in regime. After the

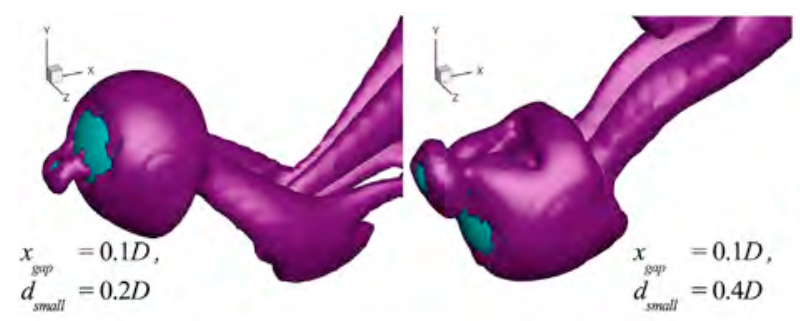

(a) (b)

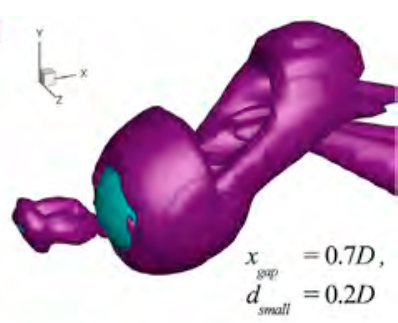

(c)

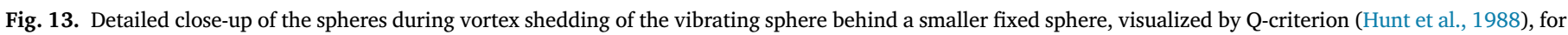
different values of $x_{\text {gap }}$ and $d_{\text {small }}$. 


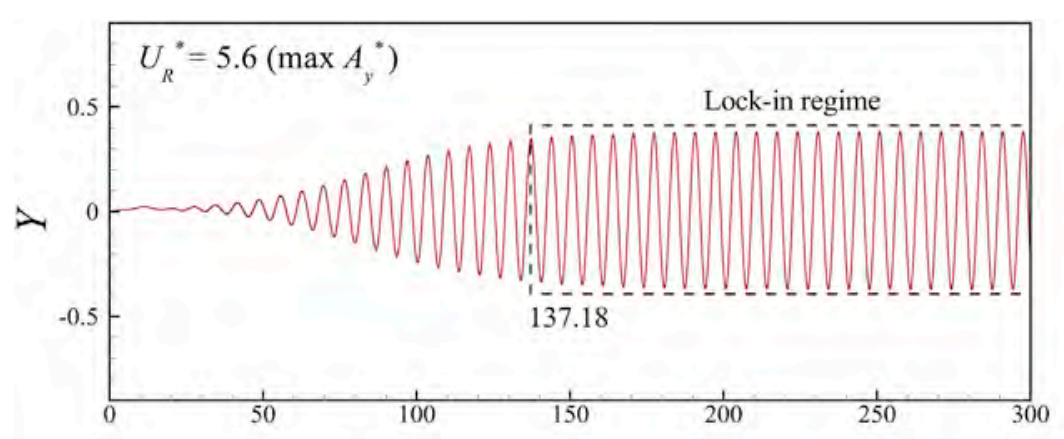

(a)

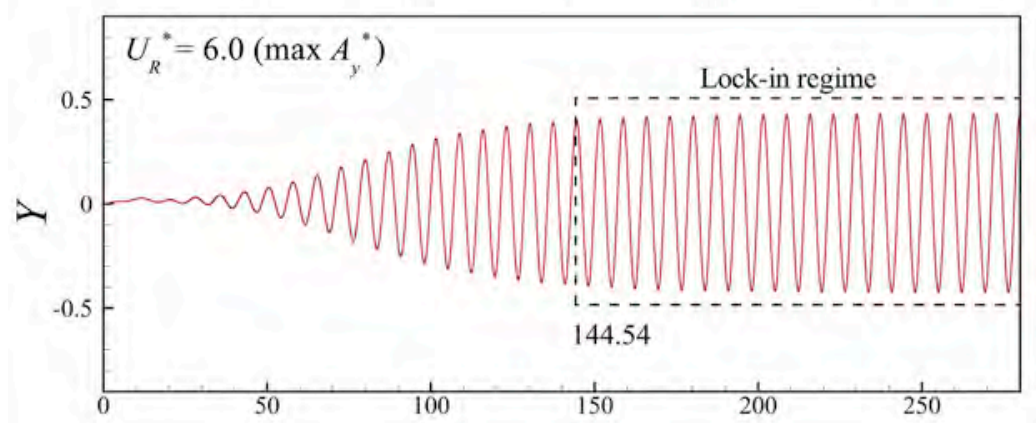

(b)

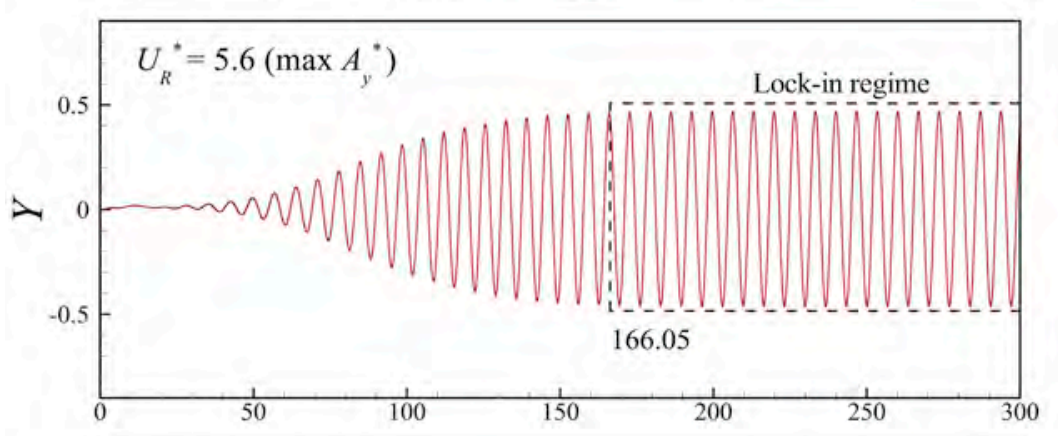

(c)

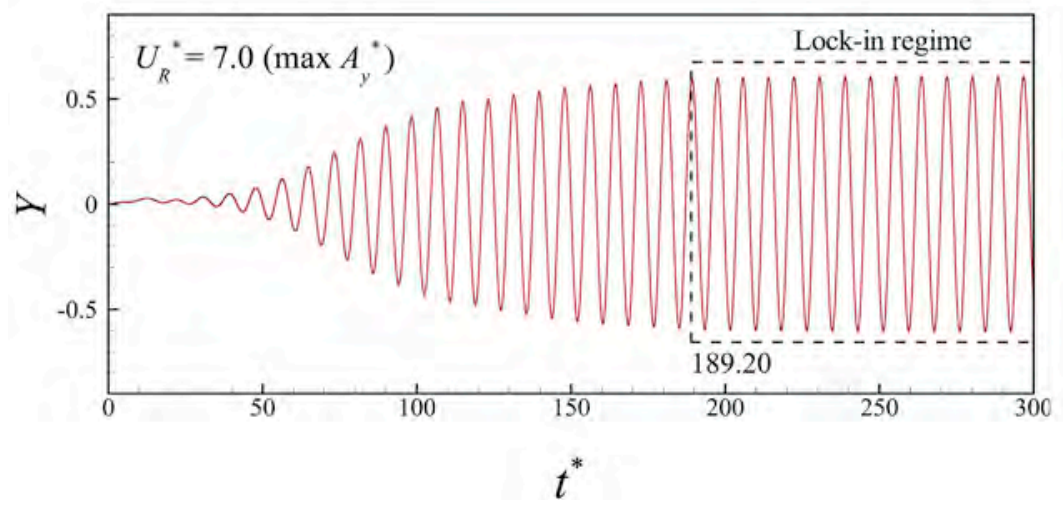

(d)

Fig. 14. Time histories of the normalized transverse displacement of the large sphere at the peak amplitude ratios for different upstream small sphere diameters; (a) $d_{\text {small }}=0.1 D$, (b) $d_{\text {small }}=0.2 D$, (c) $d_{\text {small }}=0.3 D$ and (d) $d_{\text {small }}=0.4 D$.

onset of the lock-in regime, these differences are notably smaller. A possible explanation is that before the onset of the lock-in regime, the low amplitude vibration allows the larger sphere to remain in the wake of the smaller fixed sphere, where it encounters lesser drag forces. But after the onset of the lock-in regime, the larger amplitude vibrations of the larger sphere only allow it in the wake of the smaller fixed sphere for much shorter time periods. Hence, during the lock-in regime, the average drag force on the vibrating sphere approaches closer to the drag force experienced by it when there was no fixed upstream sphere. As the influence of the lock-in regime on the vibration amplitude appears to diminish at $U_{r}^{*}=9.0$, the difference between the values of drag force coefficient for the case without the fixed sphere and cases with the fixed sphere diverges again. This highlights the limitation of the small fixed sphere in this regard, which can only influence the drag forces on the vibrating sphere at positions where the vibrating sphere is in its wake.

For the lift coefficient shown in Fig. 16(b), the values before the onset of the lock-in regime are negligible, which is why the sphere only has small amplitudes of vibration in this flow regime (see Fig. 10(a)). However, with the onset of the lock-in regime, an abrupt rise in lift coefficient to peak value is observed for all cases, with or without the upstream fixed sphere. Nevertheless, as expected, the lift coefficient is higher for the cases with the small sphere upstream. Moreover, the larger the size of the fixed sphere upstream, the larger is the lift coefficient, which naturally leads to higher amplitudes of vibration in the transverse direction, as 

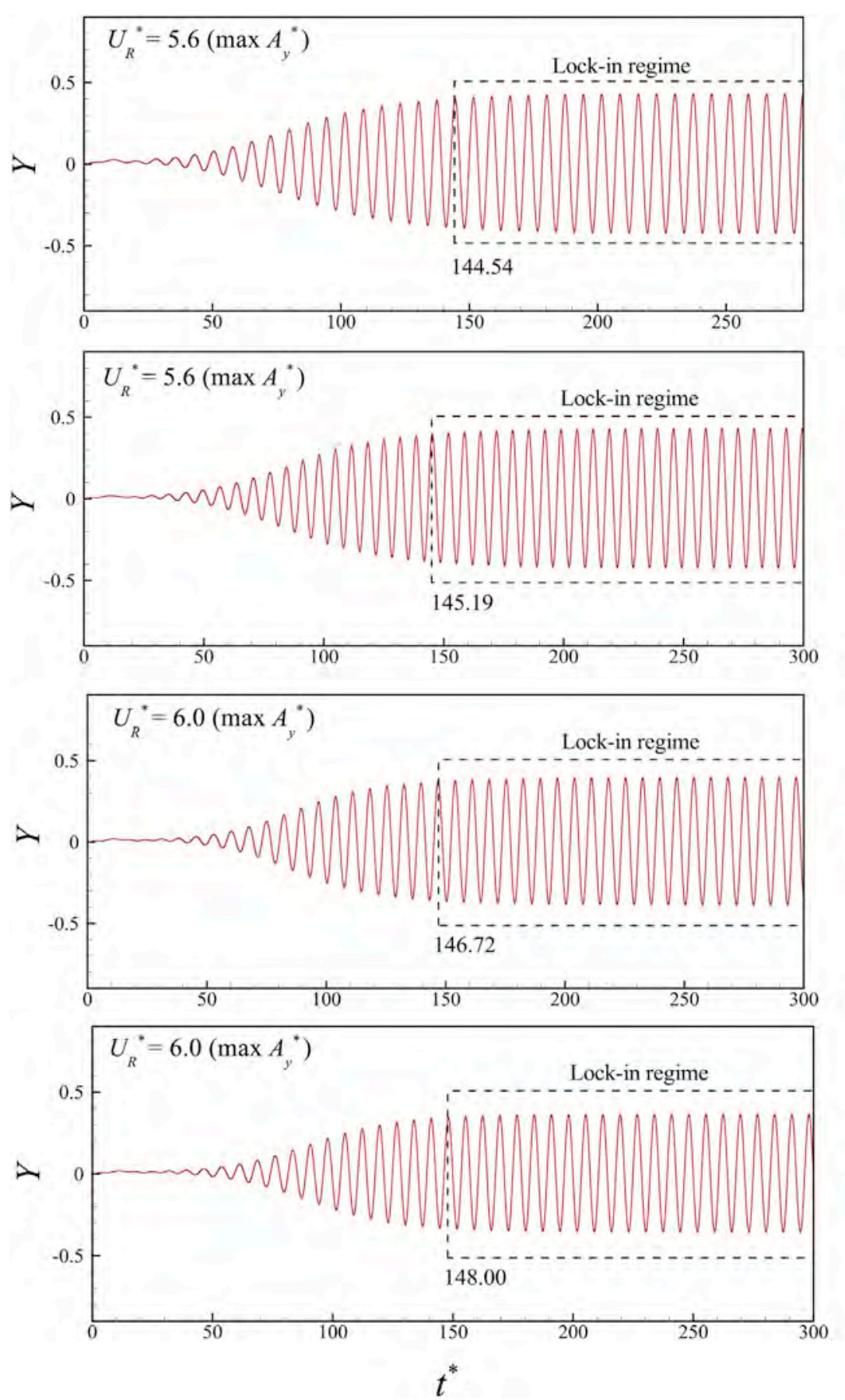

(b)

(c)

(a)

(d)

Fig. 15. Time histories of the normalized transverse displacement of the large sphere at the peak amplitude ratios for different gaps between the spheres; (a) $x_{\text {gap }}=$ $0.1 D$, (b) $x_{\text {gap }}=0.3 D$, (c) $x_{\text {gap }}=0.5 D$ and (d) $x_{\text {gap }}=0.7 D$.

discussed in section 3.3.1. This is another confirmation of the effect of the size of the upstream fixed sphere on the vibration of the larger sphere. The lift coefficient values decrease with higher reduced velocities as the lock-in region ends.

A similar trend is observed with Fig. 17(a). Before the onset of the lock-in regime, steady drag forces are observed with increasing reduced velocities, though the cases with an upstream fixed sphere have lower drag forces and the greater the gap between the sphere, the lower is the observed drag force. After the onset of the lock-in regime, the drag forces increase and then decrease with further increase in reduced velocities. However, the cases with the small sphere upstream generally exhibit lower drag forces. Moreover, even during the lock-in regime, the greater the gap between the spheres, the lower is the value of the drag force. This phenomenon may be a consequence of the fact that as we move further away from the fixed sphere, the effect of its wake disperses over a larger area and a vibrating sphere is influenced for greater time intervals when the gap between the spheres is larger.

Referring to Fig. 17(b), we observe the same negligible lift coefficients before the onset of the lock-in regime as seen in Fig. 16(b). A similar abrupt rise in lift coefficients is observed with the onset of the lock-in regime and eventually the lift coefficients decrease toward initial values as reduced velocities are increased further. However, the highest lift coefficients are observed for the case with the smallest gap between the spheres. This is consistent with the observations of Fig. 11(a) where the largest amplitudes of vibration were observed for the cases with the smallest gap between the spheres. The lift coefficients and vibration 


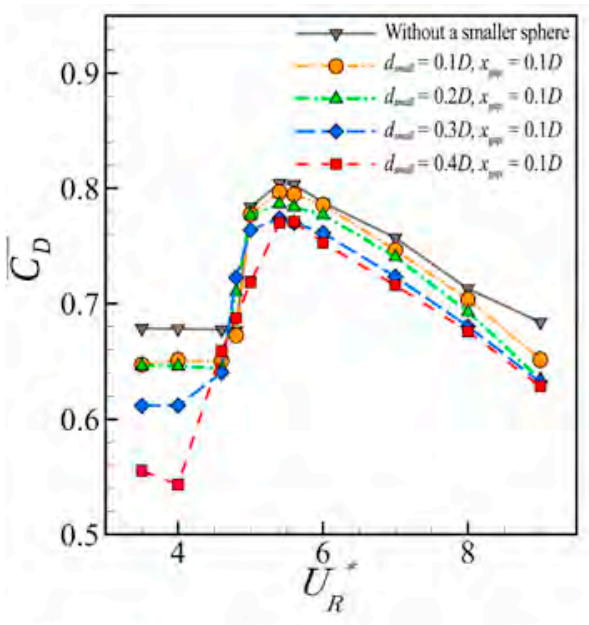

(a)

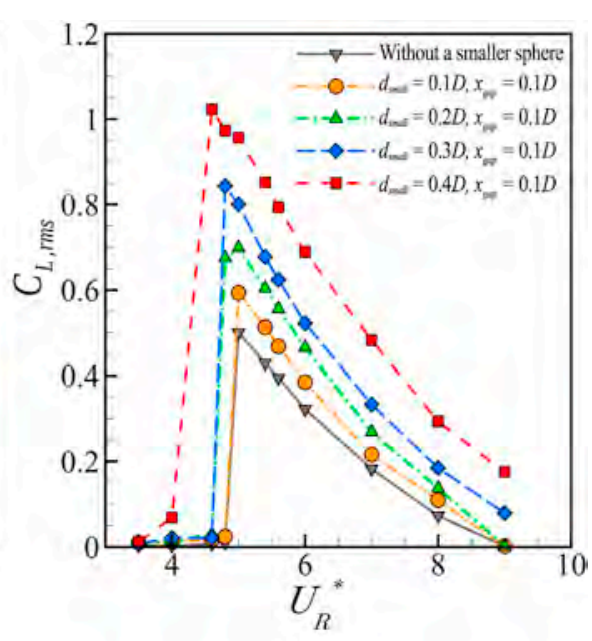

(b)

Fig. 16. Variation of (a) average drag coefficient $\left(\overline{C_{D}}\right)$ and (b) root mean square of lift coefficient $\left(C_{L, \text { rms }}\right)$ against $U_{R}^{*}$ for different values of $d_{\text {small }}$.

amplitudes of the vibrating sphere are the largest when the small fixed sphere is closest to it. Therefore, the wake of the small sphere reduces the drag forces ( $x$-direction) on the vibrating sphere while increasing the lift forces ( $y$-direction) acting upon it.

\subsection{Energy efficiency of VIV phenomena}

As the discussion of the previous sections indicates, the addition of an upstream sphere can lead to greater lift coefficients for the vibrating sphere and greater amplitudes, especially in the lock-in regime. Therefore, this could prove useful for energy conversion devices such as vortex bladeless aerogenerator and Vortex Induced Vibrations Aquatic Clean Energy (VIVACE). One of the important parameters to consider for such devices is energy efficiency, which reflects the proportion of energy that can be converted usefully through the VIV phenomenon.

The trends observed for the amplitude ratio are also observed here to a large extent, as shown in Fig. 18. For all cases, the presence of the fixed sphere upstream increases the energy conversion efficiency significantly when compared to the case without any fixed sphere. Moreover, as the size of the upstream sphere is increased, the energy efficiency increases, especially during the lock-in regime where the difference in efficiency is quite significant (see Fig. 18(a)). Moreover, the range of reduced velocities exhibiting higher efficiency is also increased for larger $d_{\text {small }}$, as for amplitude ratio shown in Fig. 10(a). On the other hand, decreasing the distance between the spheres mostly increases the energy efficiency (see Fig. 18(b)). But the highest efficiency in this figure is achieved for the case of $x_{\text {gap }}=0.3 D$ rather than $x_{\text {gap }}=0.1 D$, which suggests that there maybe an optimum value for the distance between the spheres that could be identified using a larger number of test cases. Also, there is a clear peak for energy efficiency for all tested cases occurring at $U_{R}^{*}=5.4$, which generally lies between the peak points for $A_{y}^{*}$ and $C_{L, \text { rms }}$. The highest energy efficiency that can be achieved among the tested cases using a smaller fixed sphere approaches $22 \%$ for $d_{\text {small }}=0.4 D$ and $x_{\text {gap }}=$ $0.1 D$, whereas, the maximum energy efficiency with a single sphere is only $7.2 \%$. Therefore, it can be concluded that the addition of a smaller fixed sphere upstream can enhance the energy efficiency to a large extent, in this case by almost three times.

Investigation of energy efficiency of VIV at low Reynolds numbers is lacking in published literature. Bernitsas et al. (2009) tested VIVACE device consisting of a circular cylinder in VIV at high damping and $\operatorname{Re} \approx$ $10^{5}$ and found the overall efficiency for the tested case to be $22 \%$ with a maximum peak efficiency of $30.8 \%$. For their case, they determined the theoretical upper limit of the efficiency to be $36.63 \%$.

It must be noted here that the efficiency of VIV depends on multiple

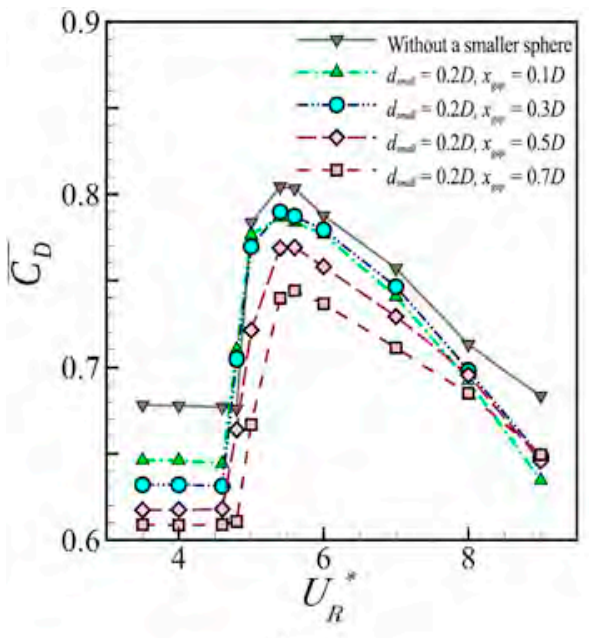

(a)

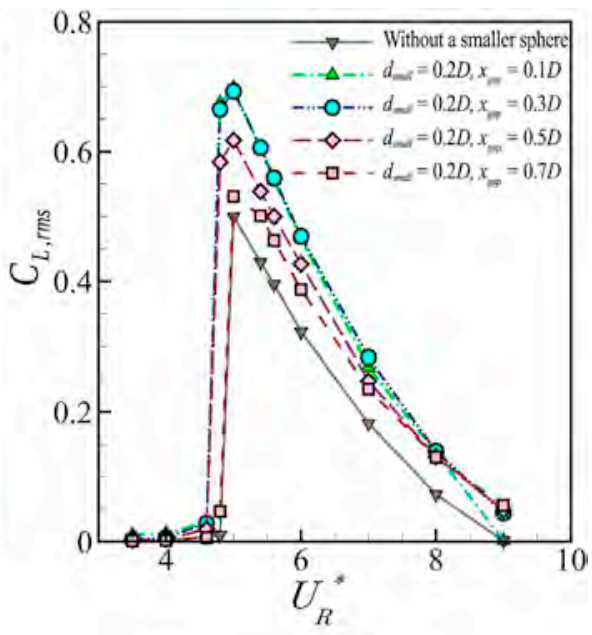

(b)

Fig. 17. Variation of (a) average drag coefficient $\left(\overline{C_{D}}\right)$ and (b) root mean square of lift coefficient $\left(C_{L, \text { rms }}\right)$ against $U_{R}^{*}$ for different values of $x_{\text {gap }}$. 


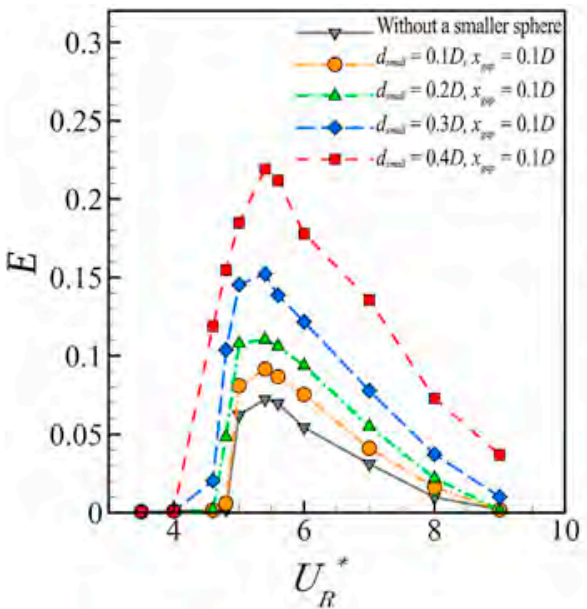

(a)

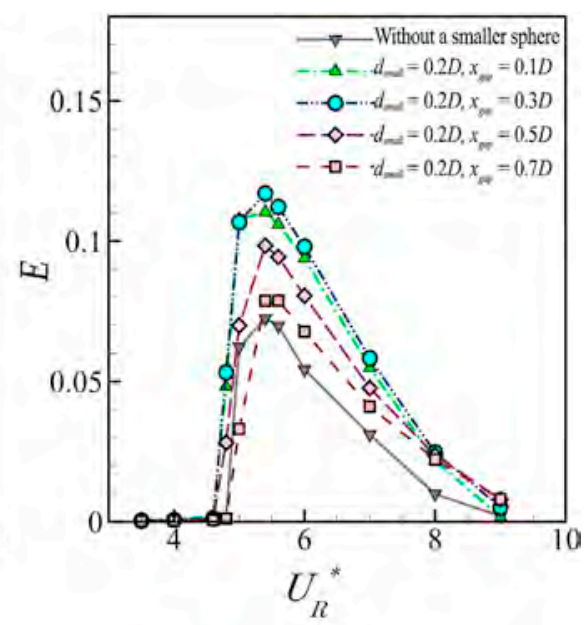

(b)

Fig. 18. Variation of energy efficiency with reduced velocity by (a) increasing the small fixed sphere diameters and (b) gap between fixed and moving sphere.
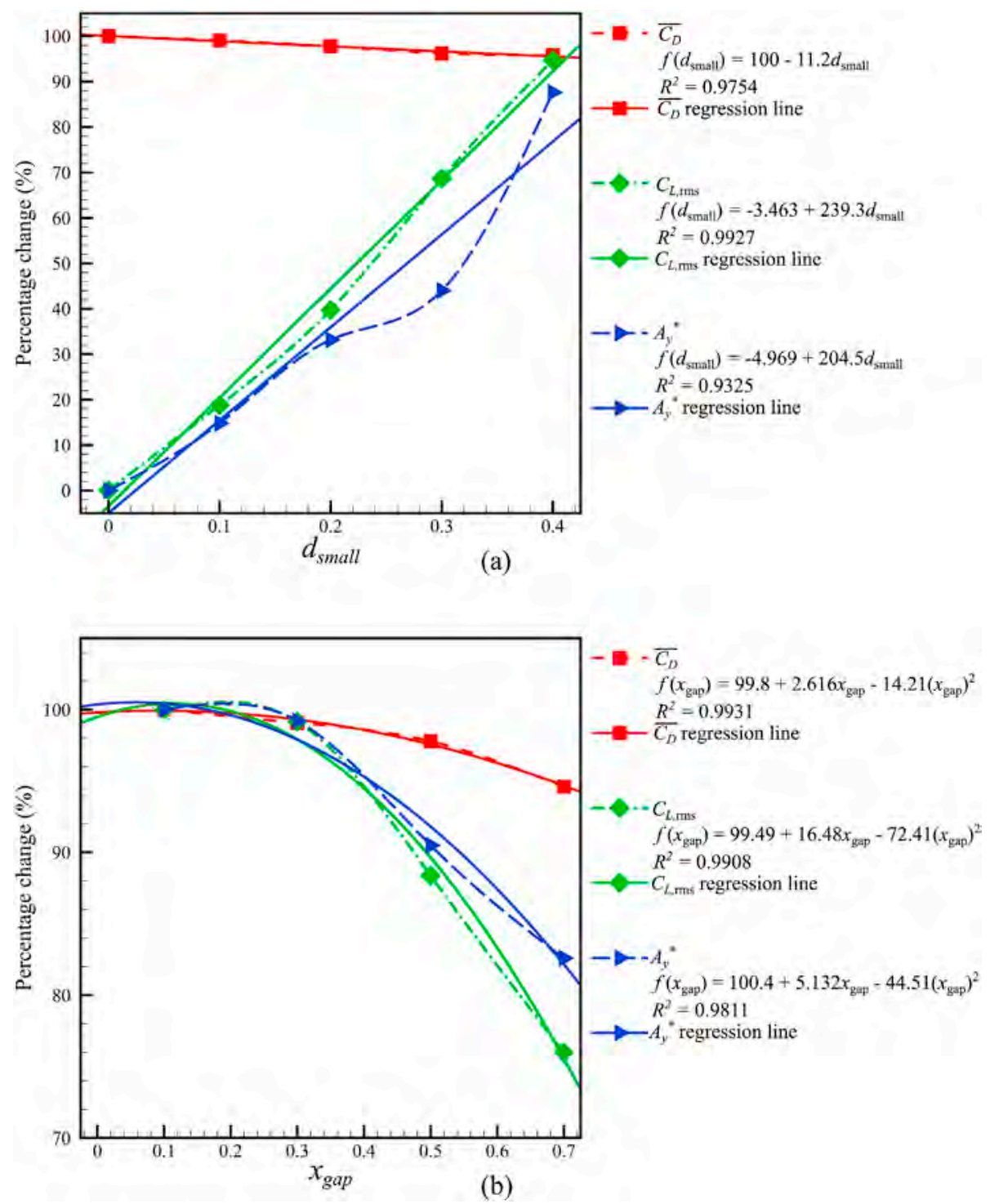

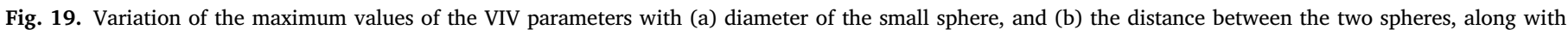
estimated regression lines. The prediction functions and values of $R^{2}$ are also shown on the figure for each of the parameters in both the cases. 
parameters including the shape, Re and structural parameters. For example, Barrero-Gil et al. (2012) conducted a parametric analysis of energy extraction from VIV (conducted for $\operatorname{Re} \geq 1000$ ) and found the energy efficiency to be dependent on mass and damping parameters as well as Reynolds number.

\subsection{Regression analysis for estimation of the effects of the small fixed sphere}

In order to investigate the effects of the small fixed sphere on the various VIV parameters of a sphere, a regression analysis was conducted based on sphere responses and force analyses.

The influence of $d_{\text {small }}$ and $x_{\text {gap }}$ on the trends of the maximum values of average drag coefficient, root mean square of lift coefficient and amplitude ratio are represented in Fig. 19. The strength of the relationship between the dependent parameters $\left(\overline{C_{D}}, C_{L, \text { rms }}\right.$ and $\left.A_{y}^{*}\right)$ and the independent variables $d_{\text {small }}$ and $x_{\text {gap }}$ can be assessed from the respective values of $R^{2}$ shown in the figure. The $R^{2}$ values listed in Fig. 19(a) indicate a strong dependence of $C_{L, \mathrm{rms}}$ on $d_{\text {small }}$ but $\overline{C_{D}}$ and $A_{y}^{*}$ are dependent on it to lesser extents, respectively. Analyzing Fig. 19(b), we notice that the dependence of all the parameters on $x_{\text {gap }}$ is of a similar nature. Moreover, the maximum values for each of the parameters can be expected to decrease as the distance between the spheres increases.

The trends shown in Fig. 19 are influenced by the size of the smaller fixed sphere and the gap between the two spheres. It should be noted that this figure only analyzes the maximum values of $\overline{C_{D}}, C_{L, \mathrm{rms}}$ and $A_{y}^{*}$. Regression line equations, which can be used to predict the maximum values are also listed for each of the parameters in both the cases.

In Table 9, the regression line equations have been tested with two randomly selected cases and the output values are compared against numerical solutions. For this purpose, another set of numerical solutions were obtained using the developed solver and the regression line equations were applied to the results of the single sphere case to conduct this comparison. From the comparison of values obtained for the two cases, the output of the regression line equations was found to be close to the results from the numerical solutions. Hence, these equations can be useful for estimating the maximum values of the parameters when a small fixed sphere is placed upstream, especially for the range of values of parameters used in this study. Further investigations may be used to improve the accuracy of the equations over a broader range.

\section{Conclusions}

The vortex-induced vibration of an elastically mounted sphere in the transverse direction is studied numerically using direct-forcing immersed boundary method for three different cases. The first case of flow past a sphere at $\operatorname{Re}=50$ is considered for the validation of the in-house numerical solver and it is found to be appropriate for usage in other cases. This is confirmed by the comparison of $\overline{C_{D}}$ and $x_{r}$ with previous studies (see Table 5).

The second case, transverse vibration of a sphere, is also a validation case but it aims to verify the solver's capability to handle VIV using DFIB method. With the same parameters and problem description as in earlier

\section{Table 9}

Comparison of the output of regression line equations with the numerical solution for two randomly selected cases.

\begin{tabular}{cllll}
\hline Case & Method & $\overline{C_{D}}$ & $C_{L, \mathrm{rms}}$ & $A_{y}^{*}$ \\
\hline \multicolumn{2}{l}{ Numerical solution } \\
1 & $d_{\text {small }}=0.15 D ; x_{\text {gap }}=0.5 D$ & 0.7417 & 0.592 & 0.366 \\
2 & $d_{\text {small }}=0.25 D ; x_{\text {gap }}=0.15 D$ & 0.7753 & 0.760 & 0.439 \\
Regression line equations & & & \\
1 & $d_{\text {small }}=0.15 D ; x_{\text {gap }}=0.5 D$ & 0.7716 & 0.593 & 0.369 \\
2 & $d_{\text {small }}=0.25 D ; x_{\text {gap }}=0.15 D$ & 0.7809 & 0.784 & 0.468 \\
\hline
\end{tabular}

studies (Behara et al., 2011; Rajamuni et al., 2018b), it is shown that the results obtained from the present study are in close agreement $\left(A_{y}^{*}, \overline{C_{D}}, C_{L, \mathrm{rms}}, f^{*}\right.$ and vortex shedding mode) with them. Based on the results of the second case of VIV problem (6), it is found that the reduced velocity $\left(U_{R}^{*}\right)$ has a large influence on flow characteristics and sphere behavior. The $f^{*}$ is close to 1 and the lift and drag force coefficients display behavior similar to those in earlier studies, thus validating the solver as well as the adequacy of the chosen model and DFIB for investigation of VIV problems.

The third case investigates the effects of a small fixed sphere in front of the vibrating sphere in tandem inline arrangement, with a variation of small sphere diameters $\left(d_{\text {small }}\right)$ and the gap between the spheres $\left(x_{\text {gap }}\right)$. Based on the sphere response, the amplitude ratio increases with $d_{\text {small }}$. This enhancement in vibration is identified as likely to have been influenced by the wake-induced vibration (WIV) caused by the smaller sphere.

The distance between the spheres is also identified as regulating the WIV phenomenon obtained from the wake of the small fixed sphere. The greater the value of $x_{\text {gap }}$, the lesser is the effect of small sphere's WIV on the downstream vibrating sphere, as seen by a decrease in amplitude ratio at larger $x_{\text {gap }}$ values. A similar trend can be observed in force analyses as the value of lift coefficient is increased by the presence of the small sphere in front of the rigid-body. The increase in lift coefficient is much greater for larger diameters of the fixed upstream sphere and it is also greater when the distance between the spheres is lesser. It is observed that the wake of the small sphere appears to enhance the lift forces acting on the vibrating sphere, leading to larger amplitudes of vibration, but diminishes the drag forces.

For diameters $d_{\text {small }} \geq 0.2 D$, the range of the lock-in regime is also increased with its onset occurring at smaller reduced velocities and continuing on for larger reduced velocities, as compared to the case without the upstream sphere. The type of vortex shedding modes do not appear to be influenced by the presence of the small fixed sphere upstream.

Up to three times higher energy efficiencies were observed with the fixed sphere upstream of the vibrating sphere. The highest energy efficiency among the tested cases was $22 \%$, which was observed for $d_{\text {small }}=$ $0.4 D$ and $x_{\text {gap }}=0.1 D$. Also, a clear peak for energy efficiency in all tested cases occurred at $U_{R}^{*}=5.4$. Further tests should be conducted to firmly establish these trends. Nevertheless, the presence of the small sphere clearly increases the energy efficiency of VIV of a downstream sphere. Finally, regression analysis was conducted to determine regression equations for the relation between the studied parameters with $d_{\text {small }}$ and $x_{\text {gap }}$, which were also tested for two random cases, though further cases are necessary to improve their accuracy.

Besides using different flow types including turbulent flow with different Reynolds numbers, future studies may also consider variations of other VIV parameters such as $m^{*}, \zeta$ as well as an extended range of $U_{R}^{*}$. The shape or arrangement of the rigid-body may also be modified to investigate whether the present conclusions for a sphere are also applicable elsewhere such as for airfoils or for vortex bladeless aerogenerator constraints. Moreover, the optimal form of shape and/or parameters may be investigated for maximizing energy efficiency.

\section{CRediT authorship contribution statement}

Syed Ahmad Raza: Conceptualization, Methodology, Software, Formal analysis, Writing - original draft, Project administration. MingJyh Chern: Conceptualization, Methodology, Software, Formal analysis, Writing - review \& editing, Supervision, Funding acquisition. Herman Susanto: Methodology, Software, Validation, Formal analysis, Investigation, Visualization. Yun-Hui Zhou: Methodology, Software, Formal analysis, Investigation, Visualization. 


\section{Declaration of competing interest}

The authors declare that they have no known competing financial interests or personal relationships that could have appeared to influence the work reported in this paper.

\section{Acknowledgments}

The authors would like to express their gratitude for the financial support from Ministry of Science and Technology, Taiwan (grant no. MOST-107-2221-E-011-075-MY3).

\section{Appendix}

\subsection{Numerical procedure}

The numerical procedure adopted in the present study is summarized here. The following steps were undertaken:

1.Apply the initial and boundary conditions

2. Calculate the value of volume of solid ( $\eta$ ) using sub-grid method.

3. Calculate the first intermediate velocity ( $\left.\mathbf{u}^{*}\right)$ using eq. (4).

4. Solve the pressure Poisson equation and advance to the second intermediate velocity ( ${ }^{* * *}$ ) using eq. (5).

5. Calculate the virtual force ( $\left.\mathbf{f}^{*}\right)$ and the flow velocities using eqs. (6) and (7).

6. Calculate the total force applied to the solid body using eq. (8).

7. Calculate the hydrodynamic force coefficients $C_{D}$ and $C_{L}$.

8. Apply the rigid-body motion equations to find the velocities and displacements of sphere(s) using eq. (9).

9. Evaluate the VIV parameters: average drag coefficient $\left(\overline{C_{D}}\right)$, root mean square of lift coefficient $\left(C_{L, \text { rms }}\right)$, frequency ratio $\left(f^{*}\right)$, amplitude ratio in the transverse direction $\left(A_{y}^{*}\right)$ and energy efficiency $(E)$.

\section{References}

Alam, M.M., Moriya, M., Takai, K., Sakamoto, H., 2003. Fluctuating fluid forces acting on two circular cylinders in a tandem arrangement at a subcritical Reynolds number. J. Wind Eng. Ind. Aerod. 1-2 (91), 139-154. ISSN 0167-6105.

Assi, G.R.S., Oct. 2014. Wake-induced vibration of tandem cylinders of different diameters. J. Fluid Struct. 50, 329-339. https://doi.org/10.1016/ j.jfluidstructs.2014.07.001. ISSN 0889-9746.

Assi, G.R.S., Meneghini, J.R., Aranha, J.A.P., Bearman, P.W., Casaprima, E., Aug. 2006. Experimental investigation of flow-induced vibration interference between two circular cylinders. J. Fluid Struct. 22 (6), 819-827. https://doi.org/10.1016/ j.jfluidstructs.2006.04.013. ISSN 0889-9746.

Assi, G.R.S., Bearman, P.W., Meneghini, J.R., Oct. 2010. On the wake-induced vibration of tandem circular cylinders: the vortex interaction excitation mechanism. J. Fluid Mech. 661, 365-401. https://doi.org/10.1017/S0022112010003095. ISSN 1469-7645.

Barrero-Gil, A., Pindado, S., Avila, S., July 2012. Extracting energy from Vortex-Induced Vibrations: a parametric study. Appl. Math. Model. 36 (7), 3153-3160. https:// doi.org/10.1016/j.apm.2011.09.085. ISSN 0307-904X.

Bearman, P.W., 1984. Vortex shedding from oscillating bluff bodies. Annu. Rev. Fluid Mech. 16 (1), 195-222. https://doi.org/10.1146/annurev.fl.16.010184.001211.

Behara, S., Sotiropoulos, F., Oct. 2016. Vortex-induced vibrations of an elastically mounted sphere: the effects of Reynolds number and reduced velocity. J. Fluid Struct. 66, 54-68. https://doi.org/10.1016/j.jfluidstructs.2016.07.005. ISSN 0889-9746.

Behara, S., Borazjani, I., Sotiropoulos, F., Nov. 2011. Vortex-induced vibrations of an elastically mounted sphere with three degrees of freedom at $\mathrm{Re}=300$ : hysteresis and vortex shedding modes. J. Fluid Mech. 686, 426-450. https://doi.org/10.1017/ jfm.2011.339. ISSN 1469-7645.

Bernitsas, M.M., Raghavan, K., 2011. Enhancement of Vortex Induced Forces and Motion through Surface Roughness Control. Patent US8047232B2, The Regents of The University of Michigan, Ann Arbor, MI, United States, Nov.

Bernitsas, M.M., Raghavan, K., Ben-Simon, Y., Garcia, E.M.H., 2008. VIVACE (vortex induced vibration aquatic clean energy): a new concept in generation of clean and renewable energy from fluid flow. J. Offshore Mech. Arctic Eng. 130 (4), Nov. https://doi.org/10.1115/1.2957913. ISSN 0892-7219.

Bernitsas, M.M., Ben-Simon, Y., Raghavan, K., Garcia, E.M.H., Feb. 2009. The VIVACE converter: model tests at high damping and Reynolds number around 105. J. Offshore Mech. Arctic Eng. 131 (1) https://doi.org/10.1115/1.2979796. ISSN 0892-7219.

Borazjani, I., Sotiropoulos, F., 2009. Vortex-induced vibrations of two cylinders in tandem arrangement in the proximity-wake interference region. J. Fluid Mech. 621, 321-364. https://doi.org/10.1017/S0022112008004850. ISSN 0022-1120.

Bourguet, R., Karniadakis, G.E., Triantafyllou, M.S., June 2011. Vortex-induced vibrations of a long flexible cylinder in shear flow. J. Fluid Mech. 677, 342-382. https:// doi.org/10.1017/jfm.2011.90. ISSN 1469-7645.

Chen, Wen-Li, Chen, Guan-Bin, Xu, Feng, Huang, Ye-wei, Gao, Dong-Lai, Li, Hui, 2020. Suppression of vortex-induced vibration of a circular cylinder by a passive-jet flow control. J. Wind Eng. Ind. Aerod. 199, 104119. https://doi.org/10.1016/ j.jweia.2020.104119. ISSN 0167-6105.

Chern, M.-J., Hsu, W.-C., Horng, T.-L., 2012. Numerical prediction of hydrodynamic loading on circular cylinder array in oscillatory flow using direct-forcing immersed boundary method. J. Appl. Math. https://doi.org/10.1155/2012/505916.
Chern, M.-J., Kuan, Y.-H., Nugroho, G., Lu, G.-T., Horng, T.-L., Nov. 2014. Direct-forcing immersed boundary modeling of vortex-induced vibration of a circular cylinder. J. Wind Eng. Ind. Aerod. 134, 109-121. https://doi.org/10.1016/ j.jweia.2014.08.015. ISSN 1676105.

Chern, M.-J., Noor, D.Z., Liao, C.-B., Horng, T.-L., Oct. 2015. Direct-forcing immersed boundary method for mixed heat transfer. Commun. Comput. Phys. 18 (4), 1072-1094. https://doi.org/10.4208/cicp.151214.250515s. ISSN 1815-2406.

Chern, M.-J., Lu, G.T., Kuan, Y.H., Chakraborty, S., Nugroho, G., Liao, C.B., Horng, T.L., Apr. 2018. Numerical study of vortex-induced vibration of circular cylinder adjacent to plane boundary using direct-forcing immersed boundary method. J. Mech. 34 (2), 177-191. https://doi.org/10.1017/jmech.2017.55. ISSN 1727-7191.

Choi, H., Jeon, W.-P., Kim, J., 2008. Control of flow over a bluff body. Annu. Rev. Fluid Mech. 40 (1), 113-139. https://doi.org/10.1146/annurev.fluid.39.050905.110149.

C. C. Feng. reportThe Measurement of Vortex Induced Effects in Flow Past Stationary and Oscillating Circular and D-Section Cylinders. PhD thesis, University of British Columbia, 1968.

Govardhan, R., Williamson, C.H.K., 1997. Vortex-induced motions of a tethered sphere. J. Wind Eng. Ind. Aerod. 69-71, 375-385. https://doi.org/10.1016/S0167-6105(97) 00170-0. ISSN 0167-6105.

Govardhan, R.N., Williamson, C.H.K., May 2005. Vortex-induced vibrations of a sphere. J. Fluid Mech. 531, 11-47. https://doi.org/10.1017/S0022112005003757. ISSN 1469-7645.

Hunt, J.C.R., Wray, A.A., Moin, P., 1988. Eddies, streams, and convergence zones in turbulent flows. In: Studying Turbulence Using Numerical Simulation Databases, 2. Proceedings of the 1988 Summer Program. Center for Turbulence Research, Stanford University, CA, United States, 193-208, CA, United States, Dec.

Ishihara, Takeshi, Li, Tian, 2020. Numerical study on suppression of vortex-induced vibration of circular cylinder by helical wires. J. Wind Eng. Ind. Aerod. 197, 104081. https://doi.org/10.1016/j.jweia.2019.104081. ISSN 0167-6105.

Johnson, T.A., Patel, V.C., Jan. 1999. Flow past a sphere up to a Reynolds number of 300. J. Fluid Mech. 378, 19-70. https://doi.org/10.1017/S0022112098003206. ISSN 1469-7645.

Khalak, Asif, Williamson, Charles H.K., 1997. Investigation of Relative Effects of Mass and Damping in Vortex-Induced Vibration of a Circular Cylinder. J. Wind Eng. Ind. Aerod. 69-71, 341-350. https://doi.org/10.1016/S0167-6105(97)00167-0. ISSN 01676105.

Leonard, B.P., June 1979. A stable and accurate convective modelling procedure based on quadratic upstream interpolation. Comput. Methods Appl. Mech. Eng. 19 (1), 59-98. https://doi.org/10.1016/0045-7825(79)90034-3. ISSN 0045-7825.

McQueen, T., Zhao, J., Sheridan, J., Thompson, M.C., Dec. 2018. Active vibration control of a sphere using rotary oscillations. Adelaide, Australia. In: Proceedings of the 21st Australasian Fluid Mechanics Conference.

McQueen, T., Zhao, J., Sheridan, J., Thompson, M.C., 2020. Feedback control of flowinduced vibration of a sphere. J. Fluid Mech. 889 (Apr), 1469-7645. https://doi.org/ 10.1017/jfm.2020.47. ISSN 0022-1120.

J. Mohd-Yusof. reportInteraction of Massive Particles with Turbulence. PhD thesis, Department of Mechanical and Aerospace Engineering, Cornell University, NY, United States, 1996.

Mysa, R.C., Kaboudian, A., Jaiman, R.K., Feb. 2016. On the origin of wake-induced vibration in two tandem circular cylinders at low Reynolds number. J. Fluid Struct. 61, 76-98. https://doi.org/10.1016/j.jfluidstructs.2015.11.004. ISSN 0889-9746. 
Parkinson, G., Jan. 1989. Phenomena and modelling of flow-induced vibrations of bluff bodies. Prog. Aero. Sci. 26 (2), 169-224. https://doi.org/10.1016/0376-0421(89) 90008-0. ISSN 0376-0421.

Peskin, C.S., Oct. 1972. Flow patterns around heart valves: a numerical method. J. Comput. Phys. 10 (2), 252-271. https://doi.org/10.1016/0021-9991(72)90065-4. ISSN 0021-9991.

Rajamuni, M.M., Thompson, M.C., Hourigan, K., July 2018a. Vortex-induced vibration of a transversely rotating sphere. J. Fluid Mech. 847, 786-820. https://doi.org/ 10.1017/jfm.2018.309. ISSN 0022-1120, 1469-7645.

Rajamuni, M.M., Thompson, M.C., Hourigan, K., Feb. 2018b. Transverse flow-induced vibrations of a sphere. J. Fluid Mech. 837, 931-966. https://doi.org/10.1017/ jfm.2017.881. ISSN 0022-1120, 1469-7645.

Rajamuni, M.M., Thompson, M.C., Hourigan, K., Mar. 2019. Vortex-induced vibration of elastically-mounted spheres: a comparison of the response of three degrees of freedom and one degree of freedom systems. J. Fluid Struct. https://doi.org/ 10.1016/j.jfluidstructs.2019.02.005. ISSN 0889-9746.

Roos, F.W., Willmarth, W.W., 1971. Some experimental results on sphere and disk drag. AIAA J. 9 (2), 285-291. https://doi.org/10.2514/3.6164. ISSN 0001-1452.

Sareen, A., Zhao, J., Lo Jacono, D., Sheridan, J., Hourigan, K., Thompson, M.C., Feb. 2018. Vortex-induced vibration of a rotating sphere. J. Fluid Mech. 837, 258-292. https://doi.org/10.1017/jfm.2017.847. ISSN 0022-1120, 1469-7645.

Sarpkaya, T., June 1979. Vortex-induced oscillations: a selective review. J. Appl. Mech. 46 (2), 241-258. https://doi.org/10.1115/1.3424537. ISSN 0021-8936.
Sarpkaya, T., May 2004. A critical review of the intrinsic nature of vortex-induced vibrations. J. Fluid Struct. 19 (4), 389-447. https://doi.org/10.1016/ j.jfluidstructs.2004.02.005. ISSN 0889-9746.

Taneda, S., Oct. 1956. Experimental investigation of the wake behind a sphere at low Reynolds numbers. J. Phys. Soc. Jpn. 11 (10), 1104-1108. https://doi.org/10.1143/ JPSJ.11.1104. ISSN 0031-9015.

A. Tomboulides, S. Orszag, and G. Karniadakis. Direct and large-eddy simulations of axisymmetric wakes. In 31st Aerospace Sciences Meeting, Reno,NV,U.S.A., Jan. 1993. American Institute of Aeronautics and Astronautics. doi: 10.2514/6.1993-546.

Violette, R., De Langre, E., Szydlowski, J., 2007. Computation of vortex-induced vibrations of long structures using a wake oscillator model: comparison with DNS and experiments. Comput. Struct. 85 (41944), 1134-1141. https://doi.org/10.1016/ j.compstruc.2006.08.005.

Willden, R.H.J., Graham, J.M.R., Apr. 2001. Numerical prediction of VIV on long flexible circular cylinders. J. Fluid Struct. 15 (3), 659-669. https://doi.org/10.1006/ ifls.2000.0359. ISSN 0889-9746.

Williamson, C., Govardhan, R., 2004. Vortex-induced vibrations. Annu. Rev. Fluid Mech. 36 (1), 413-455. https://doi.org/10.1146/annurev.fluid.36.050802.122128.

Yamamoto, C.T., Meneghini, J.R., Saltara, F., Fregonesi, R.A., Ferrari, J.A., May 2004. Numerical simulations of vortex-induced vibration on flexible cylinders. J. Fluid Struct. 19 (4), 467-489. https://doi.org/10.1016/j.jfluidstructs.2004.01.004. ISSN 0889-9746. 


\section{About the journal}

Aims and scope Editorial board Abstracting and indexing

The objective of the journal is to provide a means for the publication and interchange of information, on an international basis, on all those aspects of wind engineering that are included in the activities of the International Association for Wind Engineering http://www.iawe.org/http: //www.iawe.org/. These are: social and economic impact of wind effects; wind characteristics and structure, local wind environments, wind loads and structural response, diffusion, pollutant dispersion and matter transport, wind effects on building heat loss and ventilation, wind effects on transport systems, aerodynamic aspects of wind energy generation, and codification of wind effects.

Papers on these subjects describing full-scale measurements, wind-tunnel simulation studies, computational or theoretical methods are published, as well as papers dealing with the development of techniques and apparatus for wind engineering experiments.

Author duties: Submission of a manuscript for publication in the journal carries with it an understanding that the author, when requested, will fulfil an obligation to contribute their expertise to the review of others' manuscripts.

Benefits to authors

We also provide many author benefits, such as free PDFs, a liberal copyright policy, special discounts on Elsevier publications and much more. Please click here for more information on our https://www.elsevier.com/authors/author-servicesauthor services.

Please see our https://www.elsevier.com/journals/journal-of-wind-engineering-and-industrialaerodynamics/0167-6105/guide-for-authorsGuide for Authors for information on article submission. If you require any further information or help, please visit our https://service.elsevier.com/app/home/supporthub/publishing/Support Center

ISSN: 0167-6105

Copyright (c) 2021 Elsevier B.V. All rights reserved 


\section{About the journal}

Aims and scope Editorial board Abstracting and indexing

\section{Editor-in-Chief}

T. Stathopoulos

Concordia University, Montréal, Quebec, Canada

\section{Associate Editor}

\section{B. Blocken}

University of Technology Eindhoven, Eindhoven, The Netherlands \& KU Leuven, Belgium

\section{Editorial Board}

\section{J. Baker}

University of Birmingham, Edgbaston, Birmingham, United Kingdom

\section{C. Baniotopoulos}

University of Birmingham, Edgbaston, Birmingham, United Kingdom

C. Borri

University of Florence, Firenze, Italy

S. Y. Cao

Tongji University, Shanghai, China

1.P. Castro

University of Southampton, Southampton, United Kingdom

L Cochran

, Queensland, Australia

N.). Cook

Dorset, United Kingdom

W. A. Dalgliesh

\section{Denoêl}

University of Liege, Liege, Belgium 


\section{G. Diana}

Polytechnic of Milan, Milano, Italy

\section{R. G.J. Flay}

The University of Auckdand, Auckland, New Zealand

1. Franke

University of Siegen, Siegen, Germany

Y.J. Ge

Tongir University, Shanghai, China

K. Handa

H. Hangan

Western University, London, Ontario, Canada

R. I. Harris

, United Kingdom

I.D. Holmes

JDH Consulting, Mentone, Australia

\section{A. Kareem \\ University of Notre Dame, Notre Dame, Indiana, United States}

\section{G.A. Kopp}

Western University, London, Ontario, Canada

K. Kwok

The University of Sydney, Sydney, New South Wales, Australia

M. Matsumoto

Kyoto University, Kyoto, Japan

K. C. Mehta

National Science Foundation, Alexandria, Virginia, United States

R. N. Meroney

Colorado State University, Fort Collins, Colorado, United States of America

A. Mochida

Tohoku University, Miyagi, Japan

1. Naprstek

Crech Acaderry of Soiences, Praha, Czechia 


\section{A. Robins}

University of Surrey, Guildford, United Kingdom

P.P. Sarkar

lowa State University, Ames, lowa, United States

G. Solari

University of Genoa, Genova, Italy

Y. Tamura

Beijing Jiaotong University, Beijing, China

\section{Y. Tominaga}

Niigata Institute of Technology Faculty of Engineering Graduate School of Engineering Architecture Course, Niigata, Japan

\section{Y. Xu}

The Hong Kong Polytechnic University Department of Civil and Environmental Engineering, Hong Kong, Hong Kong

All members of the Editorial Board have identified their affiliated institutions or organizations, along with the corresponding country or geographic region. Elsevier remains neutral with regard to any jurisdictional claims. 


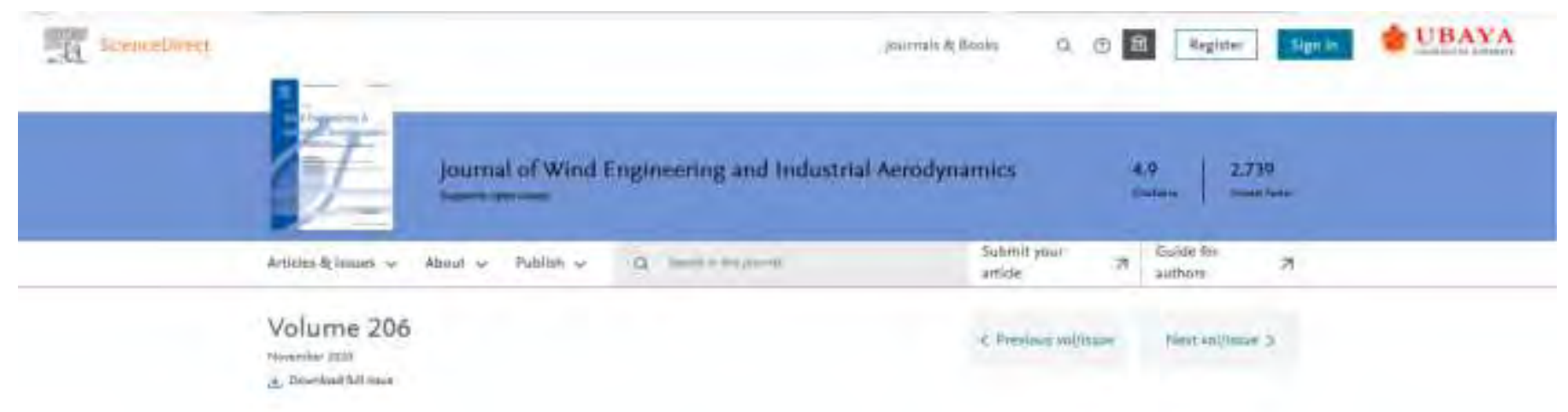

Rascarch article - Open access

How wind affects vehicles crossing a double-deck suspension bridge

Se-jin Kim, Jae-Hong Shim, Ho-Kyung Kim

Article 104329

$\Perp$ Download PDF Article preview $\checkmark$

Rescarch article - Full text access

Numerical study of the effect of aspect ratio on the flow characteristics of the Ground Transportation System

Mohab Hassaan, Diyyang Badlani, Mehdi Nazarinia

Article 104314

$\Perp$ Download PDF Article preview $\checkmark$

Rascarch articla - Full text accoss

Free-stream turbulence interaction with a wing-tip vortex

Kamal Ben Miloud, Marouen Dghim, Hachimi Fellouah, Mohsen Ferchichi

Article 104211

$\Perp$ Download PDF Article preview $\prec$

Rescarch article - Full text sccess

Analysis of turbulent structures around a rectangular prism building model using spectral proper orthogonal decomposition

Bingchao Zhang, Ryozo Ooka, Hideki Kikumoto

Article 104213

$\Perp$ Download PDF Article preview $\checkmark$

Rescarch article - Full text sccoss

Optical measurements of multi-megawatt wind turbine dynamic response Hadi Hoghooghi, Ndaona Chokani, Reza.S. Abhari

Article 104214

$\Perp$ Download PDF Article preview $\checkmark$

Roscarch articla - Full text accoss

Interference effects between two tall chimneys on wind loads and dynamic responses

Ying Sun, Zhiyuan Li, Xiaoying Sun, Ning Su, Shitao Peng

Article 104227

$\Perp$ Download PDF Article preview $\checkmark$ 
Resesrch articla - Full text sccess

Wind-induced vibration and its suppression of photovoltaic modules supported by suspension cables Xu-Hui He, Hao Ding, Hai-Quan Jing, Fang Zhang, ... Xiao-Jun Weng

Article 104275

$\Perp$ Download PDF Article preview $\checkmark$

Resesrch articla - Full text scosss

Validation of lattice Boltzmann method-based large-eddy simulation applied to wind flow around single 1:1:2 building model

Mengtao Han, Ryozo Ooka, Hideki Kikumoto

Article 104277

$\Perp$ Download PDF Article preview $\checkmark$

Rescarch article - Full text access

Side-surface openings effects on galloping instability for a rectangular cylinder

Jiaqi Wang, Tomomi Yagi, Jun Ushioda, Hidekazu Ichikawa, ... Kyohei Noguchi

Article 104303

$\Perp$ Download PDF Article preview $\vee$

Resesrch article - Full text sccess

Power generation enhancement in a horizontal axis wind turbine blade using split blades

Mohammad Moshfeghi, Nahmkeon Hur

Article 104352

$\Perp$ Download PDF Article preview $\prec$

Rasearch article - Full text sccess

RANS simulation of horizontal homogeneous atmospheric boundary layer over rough terrains by an enriched canopy drag model

Chao Li, Jinghan Wang, Gang Hu, Lixiao Li, Yiqing Xiao

Article 104281

$\Perp$ Download PDF Article preview $\checkmark$

Resesrch article - Full text access

Numerical study of turbulent flow fields in urban areas using modified $k-\varepsilon$ model and large eddy simulation

Takeshi Ishihara, Guo-Wei Qian, Yi-Hong Qi

Article 104333

$\Perp$ Download PDF Article preview $\checkmark$ 
Rescarch articla - Open access

Assessment of hybrid RANS-LES methods for accurate automotive aerodynamic simulations

P. Ekman, D. Wieser, T. Virdung, M. Karlsson

Article 104301

$\Downarrow$ Download PDF Article preview $\checkmark$

Research articls - Full text access

Experimental error examination and its effects on the aerodynamic properties of wind turbine blades Shaoning Li, Luca Caracoglia

Article 104357

$\Downarrow$ Download PDF Article preview $\vee$

Research articls - Full text access

Comparative study on the effect of aerodynamic braking plates mounted at the inter-carriage region of a high-speed train with pantograph and air-conditioning unit for enhanced braking

Jiqiang Niu, Yueming Wang, Feng Liu, Zhengwei Chen

Article 104360

$\Downarrow$ Download PDF Article preview $\vee$

Research articls - Full text access

Modified fuzzy Q-learning based wind speed prediction

Rajneesh Sharma, Tushar Shikhola, Jaspreet Kaur Kohli

Article 104361

$\Downarrow$ Download PDF Article preview $\checkmark$

Resesrch article Open access

Vertical profile characteristics of thunderstorm outflows

Federico Canepa, Massimiliano Burlando, Giovanni Solari

Article 104332

$\Downarrow$ Download PDF Article preview $\vee$

Resesrch article 0 Full text access

Observational study of wind characteristics, wind speed and turbulence profiles during Super Typhoon Mangkhut

J.Y. He, Y.C. He, Q.S. Li, P.W. Chan, ... L Li

Article 104362

$\Downarrow$ Download PDF Article preview $\vee$ 
Research article - Full text access

Effects of wind-barrier parameters on dynamic responses of wind-road vehicle-bridge system Fanrong Xue, Yan Han, Yunfeng Zou, Xuhui He, Suren Chen

Article 104367

$\Downarrow$ Download PDF Article preview $\checkmark$

Rescarch article - Full text sccess

Investigation on wind tunnel experiment of oval-shaped arch-supported membrane structures

Xiaoying Sun, Kandel Arjun, Yue Wu

Article 104371

$\Downarrow$ Download PDF Article preview $\checkmark$

Research article - Full text access

Wind tunnel measurement of overall and sectional drag coefficients for a super high-rise steel tube transmission tower

Yan Li, Zhengliang Li, Eric Savory, Yongli Zhong, Zhitao Yan

Article 104363

$\Downarrow$ Download PDF Article preview $\vee$

Research articls - Full text access

Uncertainties in prediction of local peak wind pressures on mid- and high-rise buildings by considering gumbel distributed pressure coefficients

Si Han Li, John Kilpatrick, Matthew T.L Browne, Will Yakymyk, Maryam Refan

Article 104364

$₫$ Download PDF Article preview $\checkmark$

Rssearch articla 0 Full text access

Numerical study of the effect of surface grooves on the aerodynamic performance of a NACA 4415 airfoil for small wind turbines

Yue Liu, Peifeng Li, Wei He, Kaiyong Jiang

Article 104263

$\Downarrow$ Download PDF Article preview $\checkmark$

Research article - Full text access

Three-dimensional unsteady aerodynamics of a $\mathrm{H}$-shaped vertical axis wind turbine over the full operating range

N. Franchina, G. Persico, M. Savini

Article 104273

$₫$ Download PDF Article preview $\checkmark$ 
Rascarch articla - Full text sccoss

Unsteady flow and aerodynamic behavior of high-speed train braking plates with and without crosswinds Yujia Zhai, Jiqiang Niu, Yueming Wang, Feng Liu, Rui Li Article 104309

$\Perp$ Download PDF Article preview $\checkmark$

] Rescarch articla - Full text sccoss

Experimental characterisation of the wake behind paired vertical-axis wind turbines

Antoine Vergaerde, Tim De Troyer, Sara Muggiasca, Ilmas Bayati, ... Mark C. Runacres

Article 104353

$\Perp$ Download PDF Article preview $\checkmark$

Rescarch articla - Full text accoss

Effects of sensor response and moving average filter duration on maximum wind gust measurements Amir Ali Safaei Pirooz, Richard G.J. Flay, Lorenzo Minola, Cesar Azorin-Molina, Deliang Chen Article 104354

$\Perp$ Download PDF Article preview $\prec$

$\square$ Rssasrch articla - Full text sccoss

Effects of leading-edge separation on the vortex shedding and aerodynamic characteristics of an elongated bluff body

Guiyue Duan, Shujin Laima, Wenli Chen, Hui Li

Article 104356

$\Perp$ Download PDF Article preview $\checkmark$

Rescarch article - Full text scosss

Controlling flutter of a cable-stayed bridge with output feedback driven winglets

Kamal K. Bera, Naresh K. Chandiramani

Article 104372

$\Perp$ Download PDF Article preview $\prec$

Resesrch articla - Full text accoss

Sensitivity of LES predictions of wind loading on a high-rise building to the inflow boundary condition Giacomo Lamberti, Catherine Gorlé

Article 104370

$\Perp$ Download PDF Article preview $\smile$ 
Rescarch article - Full text access

Numerical investigation of the effects of a small fixed sphere in tandem arrangement on VIV of a sphere Syed Ahmad Raza, Ming-Jyh Chern, Herman Susanto, Yun-Hui Zhou Article 104368

$\Perp$ Download PDF Article preview $\checkmark$

Rescarch article 0 Full text access

Observations of the flow experienced by a track cyclist using velodrome, wind tunnel, and potential flow investigations with an instrumented bicycle

Shaun Fitzgerald, Richard Kelso, Paul Grimshaw, Andrew Warr

Article 104374

$\Perp$ Download PDF Article preview $\checkmark$

Resesrch article - Full text sccess

Three-dimensional characteristics and axial flow pattern in the wake flow of an oblique circular cylinder Rui Wang, Dabo Xin, Jinping Ou

Article 104381

$₫$ Download PDF Article preview $\checkmark$

Resesrch article - Full text sccess

A framework for the probabilistic quantification of the resilience of communities to hurricane winds Ahmed U. Abdelhady, Seymour M.J. Spence, Jason McCormick

Article 104376

$\Perp$ Download PDF Article preview $\checkmark$

Research article - Full text access

Design of a tunnel entrance hood for high-speed trains

Sanetoshi Saito, Takashi Fukuda

Article 104375

$\Downarrow$ Download PDF Article preview $\checkmark$

Rescarch article - Full text access

CFD analysis of the aerodynamic effects on the stability of the flight of a quadcopter UAV in the proximity of walls and ground

C. Paz, E. Suárez, C. Gil, C. Baker

Article 104378

$\Perp$ Download PDF Article preview $\checkmark$ 